\title{
A new hybrid teaching-learning particle swarm optimization algorithm for synthesis of linkages to generate path
}

\author{
RAMANPREET SINGH*, HIMANSHU CHAUDHARY and AMIT K SINGH \\ Department of Mechanical Engineering, Malaviya National Institute of Technology Jaipur, Jaipur 302017, India \\ e-mail: ramanpreet.gurdutta@ hotmail.com
}

MS received 4 January 2017; revised 17 April 2017; accepted 18 April 2017; published online 7 November 2017

\begin{abstract}
This paper proposes a novel hybrid teaching-learning particle swarm optimization (HTLPSO) algorithm, which merges two established nature-inspired algorithms, namely, optimization based on teachinglearning (TLBO) and particle swarm optimization (PSO). The HTLPSO merges the best half of population obtained after the teacher phase in TLBO with the best half of the population obtained after PSO. The population so obtained is used subsequently in learner phase of TLBO. To validate the proposed algorithm, five constrained benchmark functions are considered to prove its robustness and efficiency. The proposed algorithm is applied to synthesize four-bar linkage for prescribed path. It is found that the HTLPSO performs better than other single nature-inspired algorithms for path synthesis problem in mechanism theory. Hence, HTLPSO may prove to be an important tool for mechanism design to follow the prescribed path.
\end{abstract}

Keywords. Hybrid-optimization; global optimization; PSO; TLBO; linkage; path synthesis.

\section{Introduction}

An assemblage of machine elements that produces a specified motion is known as a mechanism. The four-bar linkage is considered as a simple pin-jointed mechanism that is functionally flexible and practically important. Determination of a mechanism that fulfils certain motion specifications is kinematic synthesis [1]. It involves three areas, namely, type, number and dimensional synthesis. Kinematic synthesis can be categorized as motion, function and path generation [2, 3]. This paper deals with the dimensional synthesis of path generating mechanisms using the proposed algorithm. Various dimensional synthesis techniques such as geometrical [2], analytical [4] and numerical techniques [5] have been used. Furthermore, they are classified as precision point and optimal synthesis methods. In the precision point method, the number of desired points is limited, whereas in the optimal synthesis method there is no such limitation. Hence, it is frequently used by researchers [6-9] in comparison with other methods.

The kinematic synthesis problem using optimization methods consists of two distinct parts, namely, formulation of objective function along with the constraints and an algorithm to solve it. The Euclidean distance between the generated and desired points is the most widely used objective function [10]. Alternatively, multi-objective functions can also be used for the synthesis of path

*For correspondence generating mechanisms. For example, minimization of tracking error (TE) and transmission angle (TA) deviation from $90^{\circ}$ may be considered together for better path tracking, and minimum torque requirement [11]. Likewise, three objective functions, namely, TE, TA deviation from $90^{\circ}$ and maximum angular velocity ratio (MAVR) can also be used to improve the kinematic performance of a mechanism [12]. There are various optimization algorithms used for solving the formulated problem. 'Exact gradient method' is one such local optimization method that is free from finite-difference approximation methods, which render inaccurate results [13]. Extension of the primal-dual infeasible interior algorithm may be used for the linearly constrained convex nonlinear programming problem [14]. Another technique for the synthesis of the planar mechanism for different tasks may be exact differentiation that also improves the quality of search direction [15]. However, these calculus-based conventional local optimization techniques involve calculation of derivatives and Hessians and there also exists non-zero probability of obtaining a global optimum solution. In contrast to the classical optimization methods, nature-inspired optimization methods can be used for getting the global optimal solution [6].

The nature-inspired algorithms have become more popular for solving various non-linear problems in different fields, including path synthesis. One such nature-inspired algorithms is genetic algorithm (GA). GA can be used for solving highly nonlinear problems to find the optimum solutions and its efficiency may be increased by modifying its selection- and crossover procedure [6-8]. Alternatively, 
particle swarm optimization (PSO) and differential evolution (DE) algorithms may be used for path synthesis of four-bar linkage for tracking more than five precision points. Moreover, some refinement schemes can also be applied to the initial population that increase the computational efficiency [6]. Nature-inspired algorithms such as artificial bee colony $(\mathrm{ABC})$, the binary genetic algorithm (BGA) [9], whale optimization algorithm (WOA) [16], moth-flame optimization (MFO) [17] and simulated annealing (SA) [18] can also be used for the same purpose. However, these algorithms require tuning of algorithmic parameters; for example, GA requires crossover probability and mutation rate [19]; PSO requires inertia or weighting factor and social parameters, whereas DE needs crossover constant and scaling factor [8]. Alternatively, - optimization based on teaching-learning (TLBO) is a population-based nature-inspired optimization technique but free from initial parameters tuning. It works in two phases and updates the solution using the mean value of the population [20].

In contrast with the single nature-inspired algorithms, merging of two or three algorithms taking their distinct proficiencies can be an option to obtain efficient algorithms. Various hybridized (merged) techniques have been explored in the past for unconstrained functions [21, 22], and path-synthesis problems [22, 23]. Also, hybrid algorithms can be applied for numerous real life applications. For example, PSO-GA hybrid algorithm is used for closed-loop supply chain network design in large-scale networks to obtain superior results in comparison with GA [24]. The exploration tendencies of the biography-based optimization (BBO) are improved by incorporating the modified PSO velocity and position update mechanism of the particles [25]. This hybrid form of PSO, i.e., PSOBBO, has been applied to improve the feature selection problem in emotion and stress recognition from a speech signal. An interesting variant of PSO is used for sustainable integrated dynamic ship routing and scheduling optimization [26, 27], and maritime inventory routing to satisfy demand at different ports [28]. It is known as composite particle PSO (PSO-CP). This variant of PSO permits addressing an important issue of premature convergence. The improved sterling quality of PSO-CP helps escaping the local optima and provides near-optimal results. Another variant of PSO that is named as modified particle swarm optimization (MPSO) can also be used [29]. In this variant, improved particles (candidate solutions) are generated using the difference between the global and local best positions. Additionally, a greedy selection is also appended to this algorithm to improve its robustness. It can be applied for the form error evaluation, which contributes significantly to the production of mechanical components [29]. PSO can also be hybridized by combining with adaptive crossover and mutation rates [30] or by combining PSO with ant colony optimization (ACO) for convex and non-convex economic load dispatch (ELD) problem of a small scale thermal power system [31]. Also, a cooperative PSO (CPSO) is applied for solving function approximation and classification problems with improved accuracy [32]. This algorithm uses two swarms for the same problem (dismantled into two parts) and both the swarms work in a cooperative manner to achieve an improved solution. There are several metaheuristics available in the literature; therefore, it is important to anticipate whether more algorithms are required. The answer to this problem is presented in [33]. 'No-free-lunch' theorem has been proved to show that not all kinds of algorithms can perform equally well in solving all optimization problems [33]. This indicates that some optimizers may perform well for some set of problems while they fail to perform equally well for a different set of problems. The theorem allows the proposal of new hybrid algorithms that can solve a wider range of problems or some specific unsolved problems.

For any population-based metaheuristic optimization algorithm, the search process involves two stages, namely, exploration and exploitation stages, irrespective of their nature. The operators should be included to explore search space globally with randomized movements (as many perturbations of design variables as possible). This stage is known as exploration, where the search space is explored to get a better solution. The exploitation stage is followed by the exploration stage, which identifies and searches locally in the promising areas identified during exploration in the search space. Promoting any one of the exploration or exploitation may degrade the results of the other. A right balance between the two is required for the accurate approximation of the global optimum. This balance between exploration and exploitation is a challenging task for developing a metaheuristic algorithm because of its stochastic nature.

In this paper, a novel hybrid teaching-learning particle swarm optimization algorithm (HTLPSO) is proposed, which merges two well-established algorithms, namely, TLBO and PSO. It is observed that PSO provides results very quickly but its ability to yield the optimal solutions, especially for real life problems, is insufficient [34]. Also, the new solution replaces the old solution instead of considering the best solution, which reflects its explorative tendency, and it lacks exploitation [35]. Moreover, PSO uses the same operator for position update, which increases the probability of entrapping in local optimum [16]. Only exploration of search space may prevent an algorithm from finding accurate approximation of the optimum solution. In contrast with PSO, the TLBO algorithm incorporates both exploration and exploitation in the teaching and learning phases. The TLBO and PSO are combined in HTLPSO, which uses the explorative tendency of PSO with the explorative and exploitation tendency of teaching phaseTLBO. The combination appends the exploration of PSO and teaching-learning phases of TLBO. This is due to the use of extra updating mechanisms of swarms of PSO and learners of TLBO in HTLPSO. 
This paper proposes a novel optimization algorithm that merges the explorative tendency of PSO with the teacher phase of TLBO to generate a new population. This population is used in learner phase of TLBO and the final population obtained after learner phase ends one iteration. Hence, this algorithm is named as HTLPSO. By merging these two algorithms, the HTLPSO generates better results than either method alone. The proposed algorithm is applied for path synthesis problem of linkages. Five constrained benchmark functions are considered here to validate the algorithm proficiency. The feasibility and limitations of the new algorithm are suggested by analysing the results of the single and hybridized algorithms.

The paper is structured in six sections. The HTLPSO algorithm is presented in section 2. Section 3 validates and analyses the results of the new algorithm considering five constrained benchmark functions. The proposed algorithm is applied to the path synthesis problem of crank-rocker linkage and results are also discussed in section 4. Finally, conclusions are outlined in section 5 .

\section{Hybrid optimization algorithm}

This section proposes HTLPSO for the path synthesis problem. It is the hybridization of TLBO and PSO.

\section{$T L B O$}

TLBO is a population-based nature-inspired algorithm that does not require tuning of parameters. Parameter tuning is to find good values for the parameter before running the algorithm, and subsequently, the values remain constant for the whole process [36]. Therefore, the implementation of TLBO is the simplest among nature-inspired algorithms. It works on the philosophy of natural teaching-learning process. Here, each learner is offered a set of courses in which grades are awarded. The grade set of all learners collectively is called population set. The class is analogous to the population set in any nature-inspired algorithm while the set of courses offered to the learners is considered as design variables and numeric grades obtained by learners are considered as values of design variables. Furthermore, learners' result is akin to objective function value of optimization problem. The algorithm works in two phases, namely, teacher phase and learner phase for each iteration.

\section{Teacher phase}

Learners in the teachers' phase learn through the teacher. A teacher is considered as the most learned and knowledgeable person of a course. Therefore, learner with the best grade qualifies for the teacher. The teacher improves the mean result of his class, which depends on the quality of himself and learners. In this phase, the course grades of all learners are updated based on the course grade of the best learner, who qualified as a teacher. This step acts as the explorative tendency of the teacher phase. Subsequently, a greedy selection between initial and updated grades is exploitation. In greedy selection, learners with better results are kept along with their course grades and the new class is formed. Thus, teacher phase uses both explorative and exploitation tendencies. The teacher phase ends with the formation of a new class, which is treated as the initial population for the learner phase of the algorithm.

\section{Learner phase}

Learning is possible in two ways: one through teacher and other by mutual interaction among learners. Here, learners learn through mutual interactions. This phase starts with the new class formed at the end of teacher phase. In this phase also, both exploration and exploitation exist. In order to improve the grades, learners interact with at least one other learner in the class. The grade of the learners is improved if another learner has better grade in the corresponding course. Exploration is followed by greedy selection as in teacher phase with formation of a new class. This ends the learner phase and first iteration (teaching-learning cycle). The new class formed by greedy selection is used for next iteration in teacher phase.

Various parameters used in this algorithm are described here.

$p=$ population size, i.e., number of learners in a class

$d=$ number of design variables, i.e., number of courses offered to learners

$L_{i}, U_{i}=$ lower and upper limit on $i$ th design variable, i.e., minimum and maximum numeric grade in the $i$ th course

$t=t$ th iteration

$c=$ the number of classes in a teaching-learning cycle

$f_{j}^{c}=$ the objective function value of $j$ th learner of $c$ th class

$\mu_{i}=$ mean of $i$ th course grades of whole class

$G_{i}=$ grade of the $i$ th course whose objective function value is minimum

$g_{j i}^{q}=$ grade of the $i$ th course of the $j$ th learner and for $q$ th class, $q=1, \ldots, c$

$I=$ number of iterations

rand $=$ random number between 0 and 1

Note that class term is used here for population set $(p \times d)$.

Figure 1 shows the detailed algorithm for TLBO. In this algorithm, five classes, namely, $c_{1}, \ldots, c_{5}$, are formed in each iteration and the fifth class obtained after learner phase is treated as the first class of learners for the next iteration. A cycle of teacher-learner phase completes one iteration.

PSO

PSO is a nature-inspired algorithm and it exhibits common nature-inspired algorithm attributes such as random population initialization and optimum value search by updating generations. It is inspired by the simulation of environmental conditions. The main focus is to simulate graphically its smooth but unpredictable choreography of a bird flock. Potential solutions are called particles, which track their coordinates in the problem space corresponding to the best fitness (solution) achieved so far. The value 


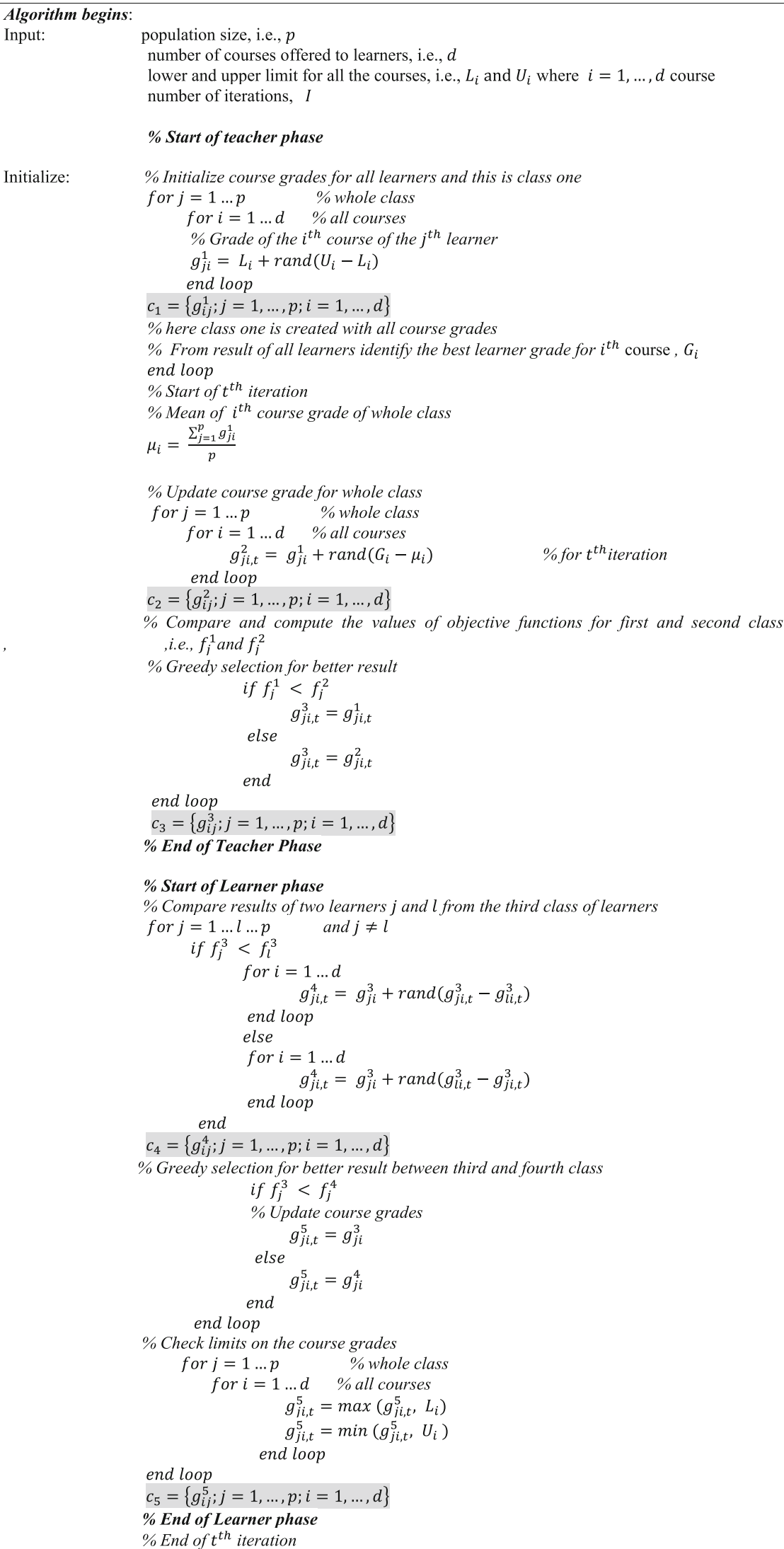

Figure 1. Algorithm for optimization based on teaching-learning. 
obtained is known as 'pBest,' while the location (value) obtained corresponding to overall best value is called 'gBest'. In PSO, velocity changes (i.e., accelerating) at each step towards its ' $p B e s t$ ' and ' $g B e s t$ ' locations. Acceleration is weighted by two separate random numbers towards 'pBest' and 'gBest' locations. Following equations are used for updating velocity and position:

$$
\begin{gathered}
V_{i+1}=w V_{i}+c_{1 a} r_{1}\left(\text { pBest }_{i}-X_{i}\right)+c_{2 a} r_{2}\left(\text { gBest }_{i}-X_{i}\right) \\
X_{i+1}=X_{i}+V_{i+1}
\end{gathered}
$$

where $V_{i+1}$ denotes a new velocity for each particle based on its previous velocity, $X_{i+1}$ denotes the updated particle's position, $r_{1}$ and $r_{2}$ are two random numbers generated in the range $[0,1]$ and $w$ is the constant parameter (inertia weight); $c_{1 a}$ and $c_{2 a}$ are the acceleration constants in which $c_{1 a}$ represents confidence of particle in itself while $c_{2 a}$ represents confidence of particle in swarm. Thus, low and high values of these constants allow the particles to flow far from the target before being pulled back and perform abrupt movement towards, or past, the target, respectively $[35,37]$. In contrast with GA, PSO does not require a special genetic operator. Here, evolution focuses only on the best solution and all the particles tend to converge to the best solution. The algorithm for PSO is shown in figure 2 .

\section{HTLPSO}

HTLPSO is a hybridized technique developed by merging PSO and TLBO. This merging makes the new algorithm useful for finding the optimum solution in a lesser number of function evaluations. The flowchart in figure 3 illustrates the HTLPSO technique. HTLPSO begins with the initialization of the population randomly. Simultaneously, initialization of PSO algorithm parameters is also done. The population so generated is treated as the initial population for teacher phase in TLBO and PSO. The best half of the population obtained in teacher phase is merged with the best half obtained in PSO. The resulting population will be of the same size as the size of the initial population. This population is used in the learner phase of TLBO, where established learner phase technique is applied. The final population obtained after learner phase will be treated as the initial population for the next iteration. The size of the final population is the same as the size of the population, which is initialized randomly. This completes one iteration of HTLPSO. This combination of established techniques takes care of the drawback of exploitation tendency of PSO and provides better results.

Various notations used for the HTLPSO algorithm are as follows:

$p_{\text {TLBO }}=$ best half of the population selected after teacher phase

$p_{P S O}=$ best half of the population selected after PSO

$p_{l}=$ best population selected for learner phase

$p_{\text {new }}=$ final population selected after each iteration

\section{Numerical examples}

In this section, validation of the proposed HTLPSO is demonstrated. The results of HTLPSO are compared to those obtained using TLBO, PSO, WOA, MFO and accelerated particle swarm optimization (APSO) technique. For PSO, $\quad c_{1}$ and $c_{2}$ are both taken as $2, \quad w=w_{\max }-$ $\left(w_{\max }-w_{\min }\right)(1 / l)$, where $w_{\max }=0.9$ and $w_{\min }=0.4$ are taken, default settings are used for other algorithms, whereas TLBO does not require algorithmic parameters. Five constrained benchmark functions are used to compare the performance of each algorithm. All these functions are to be minimized. These benchmark functions [35, 38] are defined as follows.

\section{Benchmark function 1}

minimize $f_{1}=\left(x_{1}-10\right)^{3}+\left(x_{2}-20\right)^{3}$

subject to:

$$
\begin{aligned}
& g_{1}=-\left(x_{1}-5\right)^{2}-\left(x_{2}-5\right)^{2}+100 \leq 0 \\
& g_{2}=\left(x_{1}-6\right)^{2}+\left(x_{2}-5\right)^{2}-82.81 \leq 0 \\
& 13 \leq x_{1} \leq 100, \quad 0 \leq x_{2} \leq 100 .
\end{aligned}
$$

The global minimum value of function is -6961.814 [39].

2. Benchmark function 2

$$
\begin{aligned}
& \operatorname{minimize} \\
& \qquad \begin{aligned}
f_{2}= & 5.3578547\left(x_{3}\right)^{2}+0.8356891 x_{1} x_{5}+37.293239 x_{1} \\
& -40792.141
\end{aligned}
\end{aligned}
$$

subject to:

$$
\begin{array}{rl}
g_{1}=85 & .334407+0.005685 x_{2} x_{5}+0.0006262 x_{1} x_{4} \\
+ & 0.0022053 x_{3} x_{5} \leq 0 \\
g_{2}= & -85.334407-0.005685 x_{2} x_{5}-0.0006262 x_{1} x_{4} \\
& -0.0022053 x_{3} x_{5} \leq 0 \\
g_{3}= & 80.512499+0.0071317 x_{2} x_{5}+0.002995 x_{1} x_{2} \\
& +0.0021813 x_{3}^{2}-110 \leq 0 \\
g_{4}= & -80.51249-0.0071317 x_{2} x_{5}-0.002995 x_{1} x_{2} \\
& -0.0021813 x_{3}^{2}+90 \leq 0 \\
g_{5}= & 9.300961+0.0047026 x_{3} x_{5}+0.0012547 x_{1} x_{3} \\
& +0.0019085 x_{3} x_{4}-25 \leq 0 \\
g_{6}= & -9.300961-0.0047026 x_{3} x_{5}-0.0012547 x_{1} x_{3} \\
& -0.0019085 x_{3} x_{4}+20 \leq 0 .
\end{array}
$$




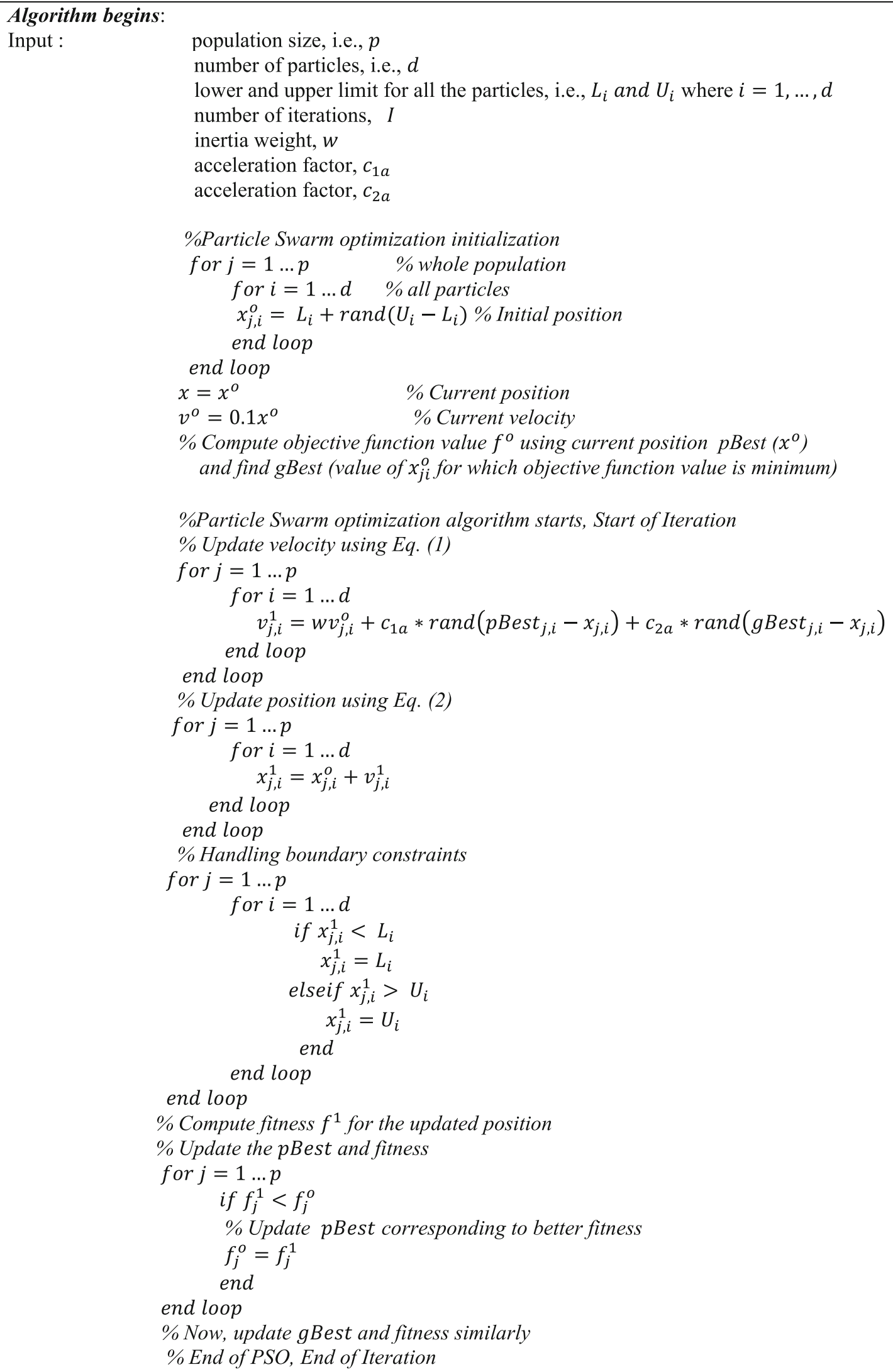

Figure 2. Algorithm for particle swarm optimization. 


$$
\begin{aligned}
& \text { Lower limit }=\left[\begin{array}{lllll}
78 & 33 & 27 & 27 & 27
\end{array}\right] . \\
& \text { Upper limit }=\left[\begin{array}{llll}
102 & 45 & 45 & 45
\end{array}\right] \text {. }
\end{aligned}
$$

The global minimum value of function is -30665.539 [39].

\section{Benchmark function 3}

\section{minimize}

$$
\begin{aligned}
f_{3}= & \left(x_{1}-10\right)^{2}+5\left(x_{2}-12\right)^{2}+x_{3}^{4}+3\left(x_{4}-11\right)^{2}+10 x_{5}^{6} \\
& +7 x_{6}^{2}+x_{7}^{4}-4 x_{6} x_{7}-10 x_{6}-8 x_{7}
\end{aligned}
$$

subject to:

$$
\begin{aligned}
& g_{1}=-127+2 x_{1}^{2}+3 x_{2}^{4}+x_{3}+4 x_{4}^{2}+5 x_{5} \leq 0 \\
& g_{2}=-282+7 x_{1}+3 x_{2}+10 x_{3}^{2}+x_{4}-x_{5} \leq 0
\end{aligned}
$$

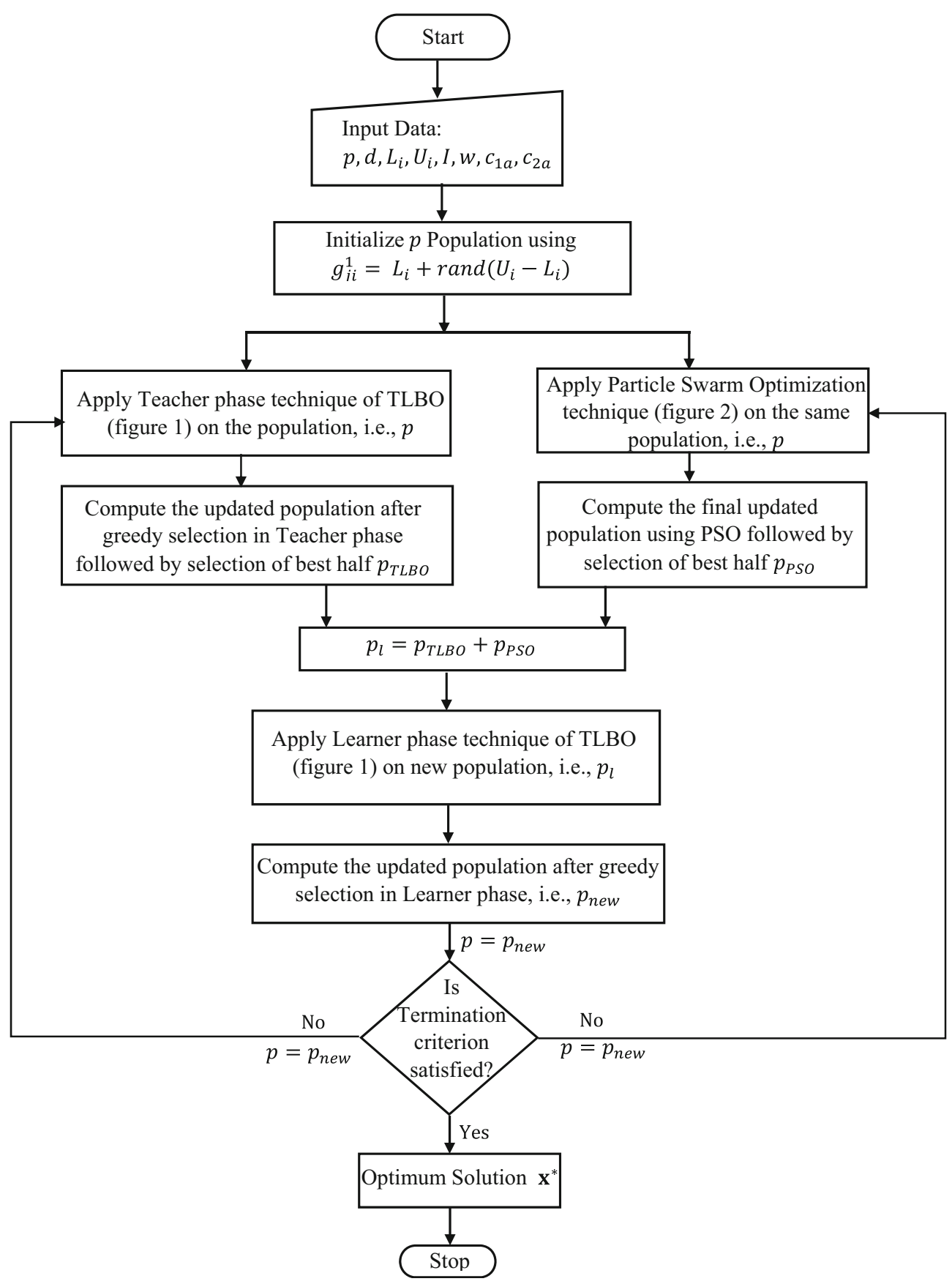

Figure 3. Flowchart of hybrid teaching-learning particle swarm optimization (HTLPSO) algorithm. 
Table 1. Performance of PSO, TLBO and HTPLSO.

\begin{tabular}{|c|c|c|c|c|c|c|c|}
\hline $\begin{array}{l}\text { Benchmark } \\
\text { function }\end{array}$ & Performance parameters & PSO & TLBO & APSO & MFO & WOA & HTLPSO \\
\hline \multirow[t]{4}{*}{1} & Best value & -6961.813 & -6961.806 & -6961.8 & -6961.736 & -6961.4234 & -6961.814 \\
\hline & Mean value & -6961.804 & -6961.778 & -6961.8 & $-6.96 \mathrm{E}+03$ & -6448.6547 & -6961.814 \\
\hline & Standard deviation & 0.00875 & 0.02388 & 0.000506 & $2.13 \mathrm{E}+02$ & $3.27 \mathrm{E}+02$ & 0.002056 \\
\hline & $\begin{array}{c}\text { Number of function } \\
\text { evaluations }\end{array}$ & 240000 & 240000 & 240000 & 240000 & 280000 & 48000 \\
\hline \multirow[t]{4}{*}{2} & Best value & -30665.54 & -30665.54 & -30666 & -30665.538 & -30668.7873 & -30665.54 \\
\hline & Mean value & -30665.38 & -30665.54 & -30666 & -30665.515 & -30234.1062 & -30665.54 \\
\hline & Standard deviation & 0.00011 & $5.561 \mathrm{E}-7$ & $4.72 \mathrm{E}-07$ & $4.49 \mathrm{E}-02$ & $2.34 \mathrm{E}+02$ & $2.283 \mathrm{E}-9$ \\
\hline & $\begin{array}{c}\text { Number of function } \\
\text { evaluations }\end{array}$ & 120000 & 240000 & 280000 & 80000 & 320000 & 60000 \\
\hline \multirow[t]{4}{*}{3} & Best value & 680.6408 & 680.6326 & 680.6308 & 680.6595 & 686.440 & 680.6308 \\
\hline & Mean value & 680.6734 & 680.6358 & 680.6668 & 680.8062 & 706.936 & 680.6334 \\
\hline & Standard deviation & 0.03060 & 0.00204 & 0.092 & 0.125689 & 22.061508 & 0.003073 \\
\hline & $\begin{array}{c}\text { Number of function } \\
\text { evaluations }\end{array}$ & 240000 & 240000 & 320000 & 120000 & 120000 & 120000 \\
\hline \multirow[t]{4}{*}{4} & Best value & -0.095825 & -0.095825 & -0.0958247 & -0.0958250 & -0.09582504 & -0.095825 \\
\hline & Mean value & -0.095825 & -0.095825 & -0.0958125 & -0.0958169 & -0.09582417 & -0.095825 \\
\hline & Standard deviation & $2.458 \mathrm{E}-8$ & $1.911 \mathrm{E}-8$ & $1.15 \mathrm{E}-05$ & $1.14 \mathrm{E}-05$ & $1.78 \mathrm{E}-06$ & $1.075 \mathrm{E}-8$ \\
\hline & $\begin{array}{c}\text { Number of function } \\
\text { evaluations }\end{array}$ & 8000 & 8000 & 28000 & 16000 & 8000 & 4800 \\
\hline \multirow[t]{4}{*}{5} & Best value & 0.000000 & $9.0381 \mathrm{E}-5$ & 0.000013 & $8.70 \mathrm{E}-05$ & $7.93 \mathrm{E}-07$ & $1.6983 \mathrm{E}-6$ \\
\hline & Mean value & $3.8680 \mathrm{E}-4$ & 0.004552 & 0.0444 & $2.16 \mathrm{E}-02$ & $1.16 \mathrm{E}-02$ & $9.6176 \mathrm{E}-4$ \\
\hline & Standard deviation & 0.00211 & 0.00407 & 0.0384 & $2.36 \mathrm{E}-02$ & 0.0195802 & 0.00143 \\
\hline & $\begin{array}{c}\text { Number of function } \\
\text { evaluations }\end{array}$ & 800 & 6400 & 6400 & 8000 & 4000 & 4800 \\
\hline
\end{tabular}

Table 2. Percentage of number of function evaluations require for HTLPSO in comparison with other algorithms.

\begin{tabular}{crrrrr}
\hline Benchmark function & PSO & TLBO & APSO & MFO & WOA \\
\hline 1 & 80 & 80 & 80 & 80 & 83 \\
2 & 50 & 75 & 79 & 25 & 82 \\
3 & 50 & 50 & 100 & 100 & 100 \\
4 & 40 & 40 & 70 & 70 & 40 \\
5 & 180 & 25 & 40 & 40 & 20 \\
\hline
\end{tabular}

$$
\begin{gathered}
g_{3}=-196+23 x_{1}+x_{2}^{2}+6 x_{6}^{2}-8 x_{7} \leq 0 \\
g_{4}=4 x_{1}^{2}+x_{2}^{2}-3 x_{1} x_{2}+2 x_{3}^{2}+5 x_{6}-11 x_{7} \leq 0 \\
\text { where } \quad-10 \leq x_{i} \leq 10 \quad(i=1, \ldots, 7) .
\end{gathered}
$$

The global minimum value of function is 680.63 [39].

4. Benchmark function 4

$$
\operatorname{minimize} f_{4}=-\frac{\sin x^{3}\left(2 \pi x_{1}\right) \sin \left(2 \pi x_{2}\right)}{x_{1}^{3}\left(x_{1}+x_{2}\right)}
$$

subject to:

$$
g_{1}=x_{1}^{2}-x_{2}+1 \leq 0
$$

$$
g_{2}=1-x_{1}+\left(x_{2}-4\right)^{2} \leq 0
$$

where $0 \leq x_{i} \leq 10(i=1,2)$.

The global minimum value of function is -0.095825 [35].

5. Benchmark function 5 [38]

minimize $f_{5}=\left(x_{1}^{2}+x_{2}-11\right)^{2}+\left(x_{1}^{2}+x_{2}^{2}-7\right)^{2}$

subject to:

$$
\begin{gathered}
g_{1}=26-\left(x_{1}-5\right)^{2}-x_{2}^{2} \geq 0 \\
\qquad g_{2}=20-4 x_{1}-x_{2} \geq 0 \\
\text { where } \quad-5 \leq x_{1} \leq 10, \quad-10 \leq x_{2} \leq 5
\end{gathered}
$$

The global minimum value of the function is 0 [38].

The performance of any new optimization algorithm on the benchmark functions should be checked with known global optima. Therefore, the same practice is adopted here to test the performance of HTLPSO algorithm. Since the metaheuristics are stochastic optimization techniques, for each benchmark function, the HTLPSO algorithm is run 30 times to obtain reasonable results. The same process is followed for all the algorithms used in this study. The 


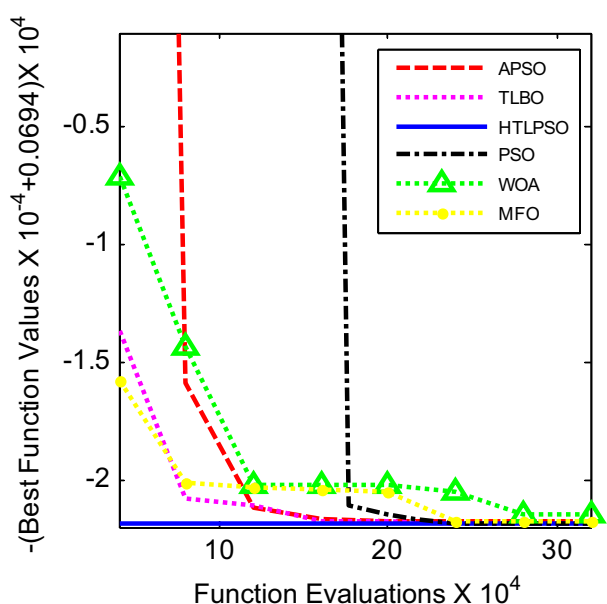

(a)

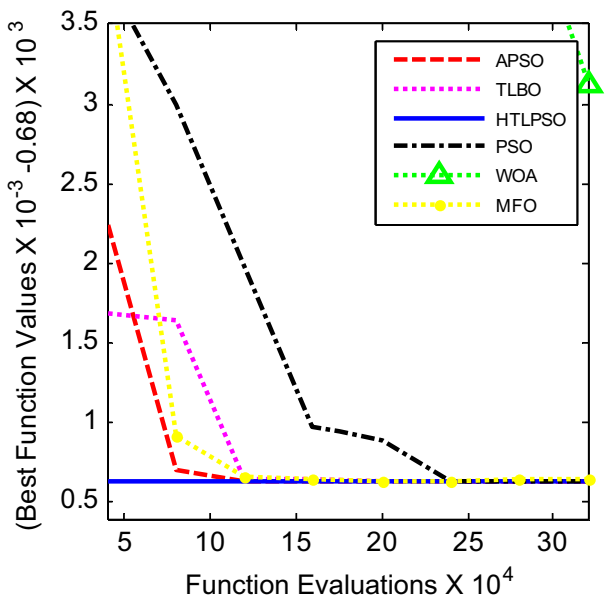

(c)

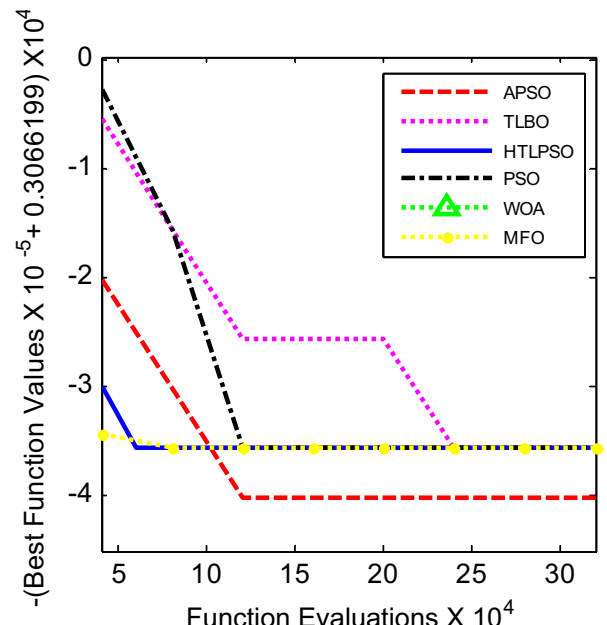

(b)

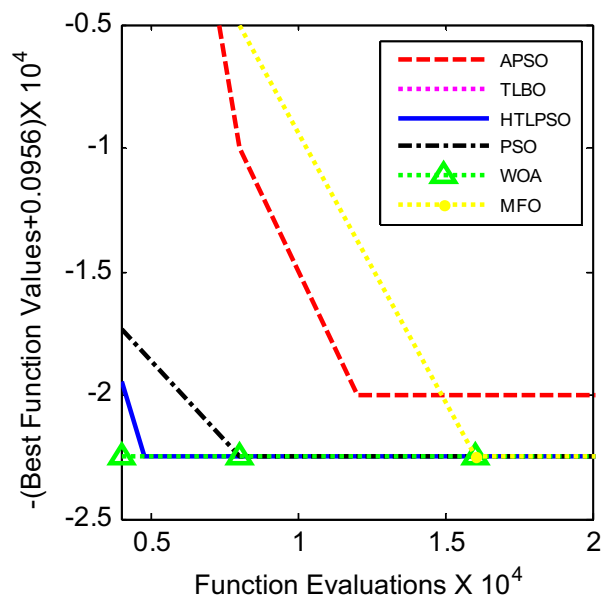

(d)

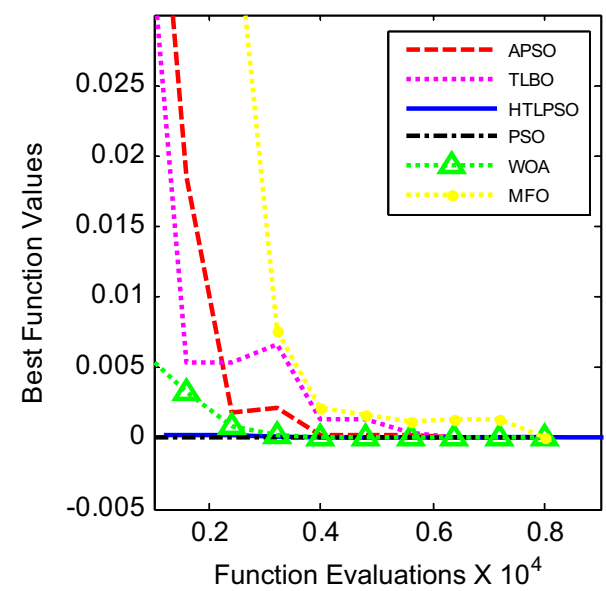

(e)

Figure 4. Convergence of the best objective function values with respect to the number of function evaluations for (a) benchmark 1, (b) benchmark 2, (c) benchmark 3, (d) benchmark 4 and (e) benchmark 5. 


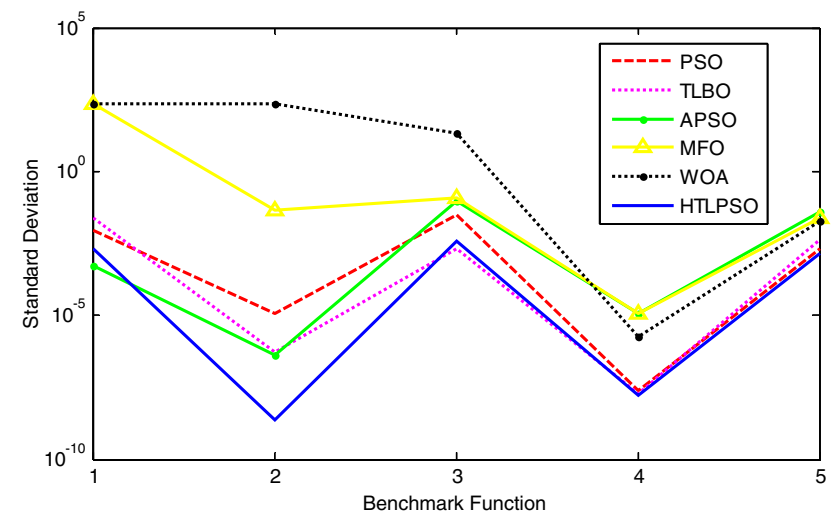

Figure 5. Comparison of standard deviation for all benchmark functions using different algorithms.

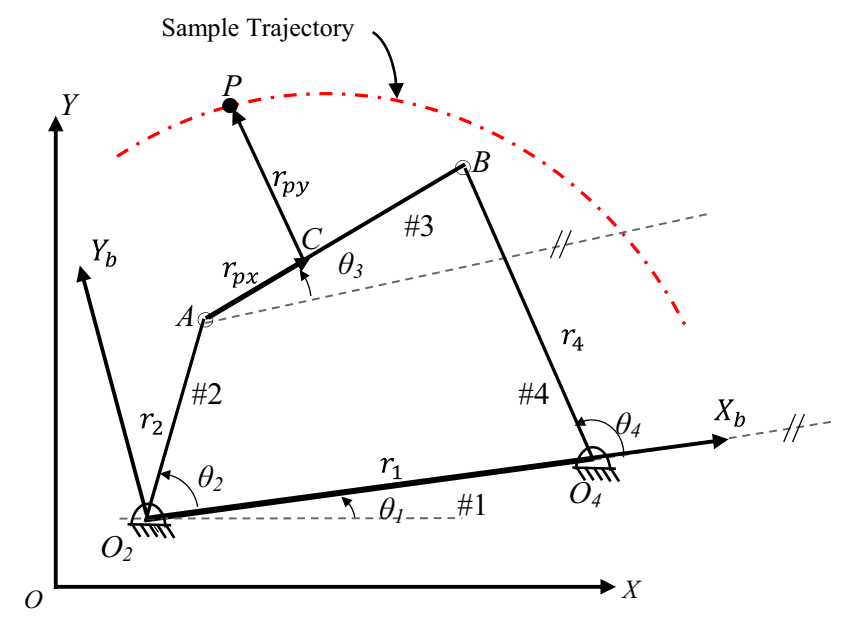

Figure 6. Four-bar linkage and definition of various notations.

objective function value corresponding to each iteration is recorded. To verify the performance of HTLPSO, its results are compared with the well-established single, nature-inspired, modified variant, and hybrid algorithms. Table 1 summarizes the statistical results (i.e., the average objective function value and corresponding standard deviation) for HTLPSO and other algorithms used in this study to solve the benchmark problems. It is observed that the HTLPSO algorithm outperforms all the other algorithms in terms of efficiency (ability to locate the global optimum in less number of function evaluations) for four benchmark functions; however, the HTLPSO takes more function evaluations for the fifth benchmark function to reach global optimum but provides best mean results. A reduction of $17-80 \%$ in the number of function evaluations is observed in the comparison of other algorithms as shown in table 2. These superior results are obtained through the combination of two well-established algorithms. The combination appends the exploration of PSO and teaching-learning phases of TLBO. This is due to the use of extra updating mechanisms of swarms of PSO and learners of TLBO used in HTLPSO, as shown in figures 1 and 2, respectively. These updating mechanisms of teaching phase widen the exploration in the search space. However, only exploration may avoid reaching the optimal solution; therefore, it is followed by exploitation (greedy selection of best solutions), which identifies the promising areas of the solution. PSO operator of updating mechanism is applied in parallel to further explore the area for the optimal solution but PSO lacks exploitation; therefore, to widen the TLBO search capability and to compensate the absence of exploitation in PSO, both these algorithms are combined. Hence, the search space is explored widely and refined; thereafter, the best half populations from both the results are selected. The well-established learning technique is then applied to the selected population to explore and exploit the search space to find the accurate approximation of the optimal solution. In this way, a right balance between the exploration and exploitation is created, which gives superior results in comparison with the single nature-inspired algorithms used here.

In order to illustrate the results lucidly, the best objective function values are plotted against the number of function evaluations for all the benchmark functions as shown in figure 4. It is observed that HTLPSO's learners and swarms tend to explore the promising regions of search space, simultaneously, and exploit the best region. Initially, the population (learners and swarms) changes abruptly and thereafter converges to an optimum point. This behaviour of the population-based algorithms is shown by the convergence curve in figure 4. It is found that HTLPSO is more competitive in comparison with all the other wellestablished metaheuristic algorithms.

From figure 4, three different trends in convergence are observed. Firstly, the convergence of the algorithm occurs rapidly after initial steps of iterations. This behaviour is seen for the HTLPSO (benchmark functions-(1-5)), WOA (benchmark functions- $(4,5)$ ), MFO (benchmark functions$(2,3)$ ), PSO (benchmark function-(5)), APSO (benchmark function-(3)) and TLBO (benchmark function-(3)). Only HTLPSO shows this trend in the convergence of the best values of the objective function in all the benchmark functions because of good balance of exploration and exploitation that helps HTLPSO to find the global minimum. This also ensures the high success rate of HTLPSO in solving the wide range of problems. Secondly, the convergence of algorithm occurs when the maximum number of function evaluations is reached. This trend is observed for the WOA (benchmark functions-(1-3)), MFO (benchmark functions-(1, 4, 5)), PSO (benchmark functions- $(1,3)$ ) and TLBO (benchmark functions- $(2,4)$ ). The convergence trend may be due to the failure of the algorithm in finding the promising solution for exploitation in the initial steps. Therefore, algorithms keep on exploring the search space to find good solutions. The third case is when the algorithm converges in an accelerated fashion. This trend is observed 
in the APSO (benchmark functions-(1, 2, 4, 5)), PSO $(2,4)$ and TLBO $(1,5)$. This behaviour is observed due to the operators used in the algorithm, which help in exploring the promising regions of the search space, thereafter converging rapidly after almost half of the maximum number of the function evaluations. Overall, it is found that HTLPSO converges fast in comparison with the other algorithms used here.

Figure 5 shows standard deviation of the objective function value for all the benchmark functions using six metaheuristic algorithms. It is found that standard deviation for HLPSO is the least in comparison with all the other algorithms. This shows that the solution obtained by HTLPSO is more reliable than the other algorithms used here.

\section{Application-path synthesis of four-bar linkage}

The HTLPSO developed in section 2 is applied to solve a four-bar path synthesis problem. Figure 6 shows various design parameters of a four-bar linkage. $X O Y$ represents a global reference frame; a coupler point $P$ is defined by vectors $\overrightarrow{A C}$ and $\overrightarrow{C P}$, where $\overrightarrow{A C}$ is along $\overrightarrow{A B}$ and $\overrightarrow{C P}$ is normal to $\overrightarrow{A B}$ and passing to point $P$. The length between joints is used to define link length as shown in figure 6. A frame $X_{b} O_{2} Y_{b}$ is defined to measure the orientation of links with respect to link 1 . It is fixed to link \#1 and its $X$-axis is aligned along link \#1. Now, orientations of link \#2, link \#3 and link \#4 are given by angles $\theta_{2}, \theta_{3}$ and $\theta_{4}$, respectively, with respect to the axis $X_{b}$. The orientation of link \#1 is defined by angle $\theta_{1}$ with respect to the fixed axis $X$. The coordinates of coupler point $\mathrm{P}$ in frame $\mathrm{X}_{b} \mathrm{O}_{2} Y_{b}$ are given as follows:

$$
\begin{gathered}
P_{x b}=r_{2} \cos \theta_{2}+r_{p x} \cos \theta_{3}-r_{p y} \sin \theta_{3}, \\
P_{y b}=r_{2} \sin \theta_{2}+r_{p x} \sin \theta_{3}+r_{p y} \cos \theta_{3} .
\end{gathered}
$$

The coupler point coordinates in fixed reference frame $X O Y$ are obtained as follows:

$$
\begin{aligned}
& P_{x}=O_{2 x}+P_{x b} \cos \theta_{1}-P_{y b} \sin \theta_{1} \\
& P_{y}=O_{2 y}+P_{x b} \sin \theta_{1}+P_{y b} \cos \theta_{1}
\end{aligned}
$$

where $O_{2 x}$ and $O_{2 y}$ are the coordinates of the $O_{2}$ in the frame $\mathrm{XOY}$.

Equations (5) and (6) represent the coordinates of any point on the path traced by the coupler point $P$. Link lengths $r_{1}, r_{2}, r_{3}$ and $r_{4}$, orientation $\theta_{1}$ of link\#1, $N$ positions of link\#2 defined by $\theta_{2}^{1}, \theta_{2}^{2}, \ldots, \theta_{2}^{N}$ and lengths $A C\left(r_{p x}\right)$ and $C P\left(r_{p y}\right)$ are to be determined for prescribed trajectory of point $P$. These $N+9$ unknown parameters are considered as design variables to synthesize the linkage:

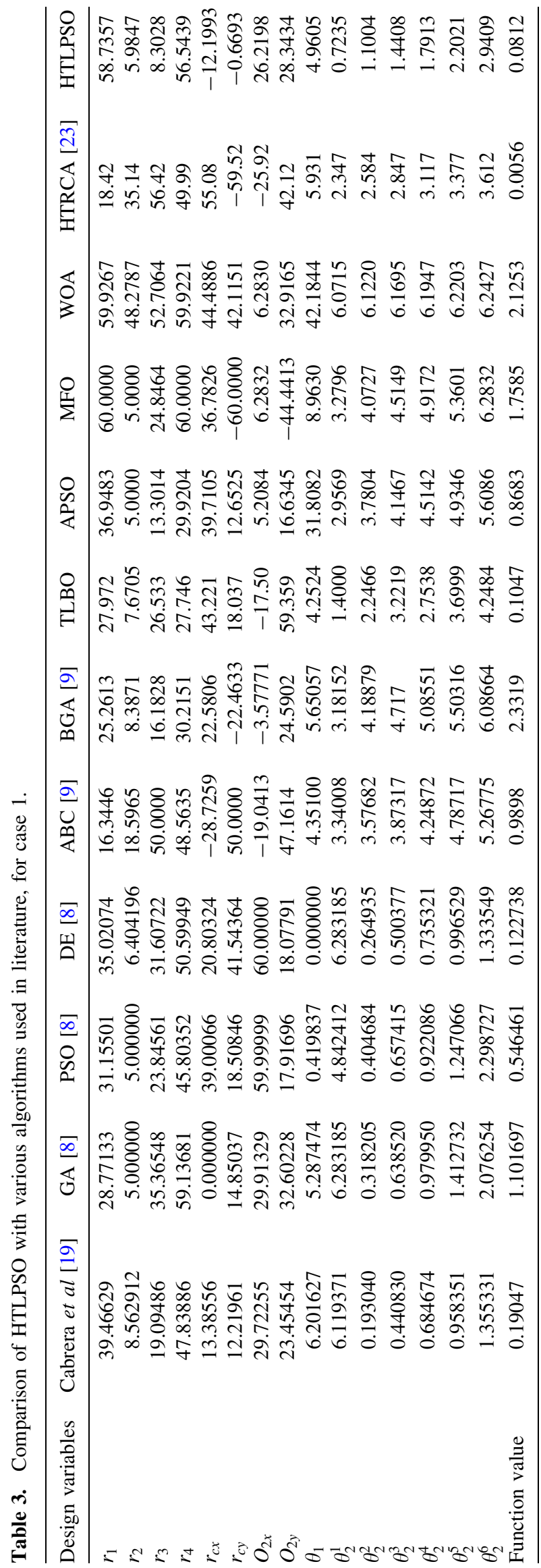




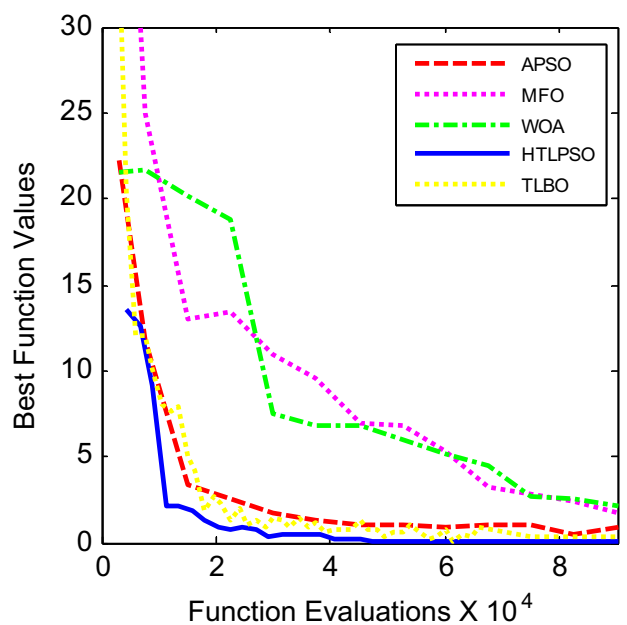

(a)

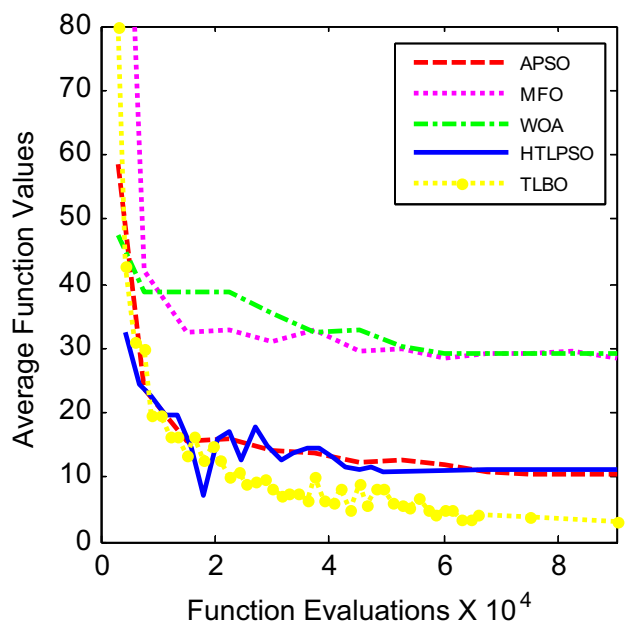

(b)

Figure 7. Convergence between (a) best function values and number of function evaluations and (b) average function values and number of function evaluations for case 1.

$$
\mathbf{x}=\left[r_{1}, r_{2}, r_{3}, r_{4}, r_{p x}, r_{p y}, O_{2 x}, O_{2 y}, \theta_{1}, \theta_{2}^{1}, \theta_{2}^{2}, \ldots, \theta_{2}^{N}\right]
$$

where $N$ represents the number of desired points for the coupler trajectory, and the design variables are defined in figure 6 . The other parameters $\theta_{3}$ and $\theta_{4}$ can be determined by loop closure equation of the mechanism [8].

Normally, for path generation problem, the position error is considered as the square root of the sum of the square of the Euclidean distances between each coupler-point position $P$ and the corresponding desired point $P_{d}$. Then, the position error is the objective function to be minimized for the synthesis problem and is expressed as follows:

$$
f(\mathbf{x})=\sum_{k=1}^{N} \sqrt{\left(P_{x}^{k}-P_{x d}^{k}\right)^{2}+\left(P_{y}^{k}-P_{y d}^{k}\right)^{2}}
$$

where $P_{x d}, P_{y d}$ are the $x$ and $y$ coordinates of desired points and $P_{x}, P_{y}$ are the $x$ - and $y$-coordinate of the coupler point trajectory. Superscript $k$ represents the $k^{\text {th }}$ position of the coupler.

A four-bar linkage is expected to achieve this objective, such that crank is able to rotate completely in sequence, either clockwise or counter-clockwise, and values of design variables should lie within the prescribed range. The following constraints are imposed to get feasible four-bar linkage.

(a) Grashof constraint:

$$
g_{1}(\mathbf{x})=r_{1}+r_{2}-r_{3}-r_{4}<0 \quad \text { if } \quad\left(r_{2}<r_{3}<r_{4}<r_{1}\right) .
$$

(b) Order constraint:

$$
g_{2}(\mathbf{x})=\theta_{2}^{k}-\theta_{2}^{k+1}<0 \quad k=1, \ldots, N .
$$

(c) Range of variables:

$$
L_{i} \leq x_{i} \leq U_{i}
$$

where $L_{i}$ and $U_{i}$ are the lower and upper limits for the $i$ th design variable. Thus using, Eqs. (7)-(10), the problem is completely stated as follows:

$$
\begin{array}{cl}
\text { minimize } & f(\mathbf{x})=\sum_{k=1}^{N} \sqrt{\left(P_{x}^{k}-P_{x d}^{k}\right)^{2}+\left(P_{y}^{k}-P_{y d}^{k}\right)^{2}} \\
\text { subject to } & g_{1}(\mathbf{x})=r_{1}+r_{2}-r_{3}-r_{4}<0 \text { if }\left(r_{2}<r_{3}<r_{4}<r_{1}\right) \\
& g_{2}(\mathbf{x})=\theta_{2}^{k}-\theta_{2}^{k+1}<0 \quad k=1, \ldots, N \\
& L_{i} \leq x_{i} \leq U_{i}
\end{array}
$$

Now, the constrained optimization problem defined by Eq. (11) is transformed into an unconstrained problem for obtaining a feasible and optimum solution. This is achieved by penalizing the objective function for any constraint violation. A large value of order $10^{4}$ is added to the objective function, which assures that infeasible solution has function value greater than the feasible solution. This eliminates all the infeasible solutions and promotes the algorithm convergence towards the optimum solution. Finally, the original problem is transformed into an unconstrained problem, which is expressed as follows:

minimize

$$
f(\mathbf{x})=\sum_{k=1}^{N} \sqrt{\left(P_{x}^{k}-P_{x d}^{k}\right)^{2}+\left(P_{y}^{k}-P_{y d}^{k}\right)^{2}}+\sum_{m=1}^{2} B_{m}\left(P^{m}\right)
$$

$$
\text { range of variables } L_{i} \leq x_{i} \leq U_{i}
$$

where $P^{m}(m=1,2)$ are the constants with value $\left(10^{4}\right)$ for penalizing the objective function if the constraints are 


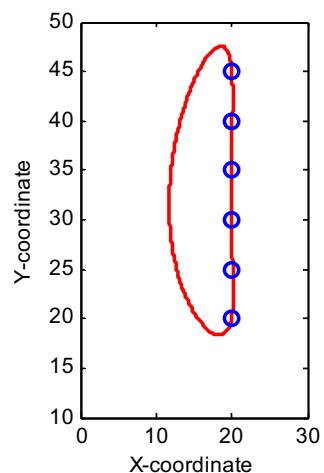

Result from literature [17]

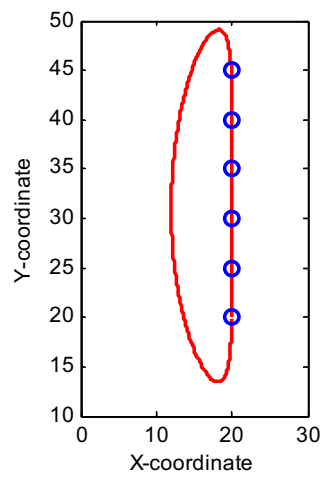

DE [14]

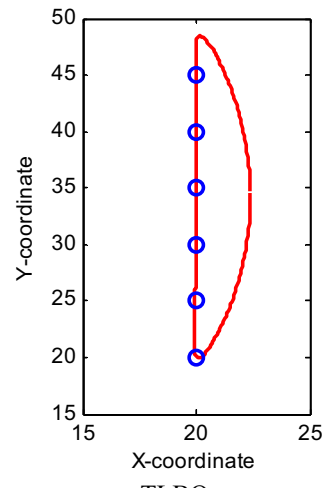

TLBO

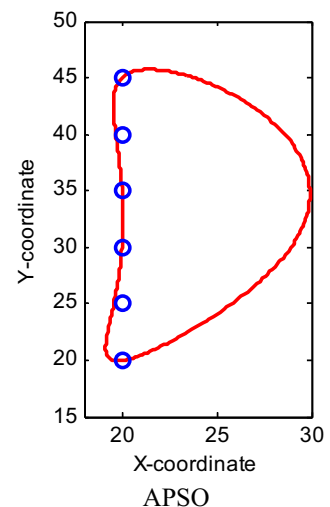

APSO

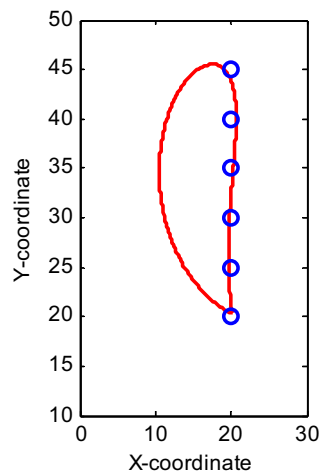

GA [14]
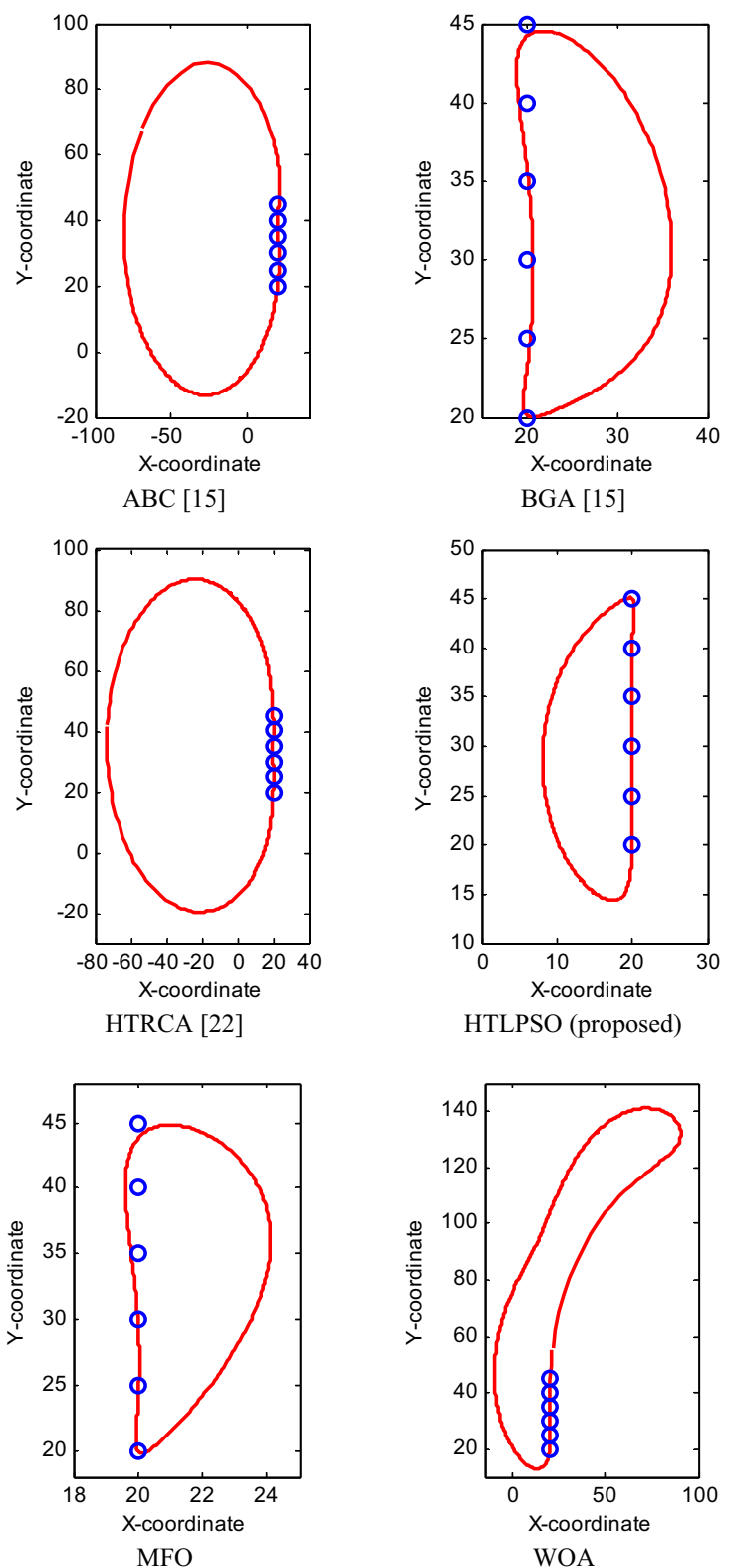

\section{Prescribed Points}

Figure 8. Generated trajectory and prescribed points. 


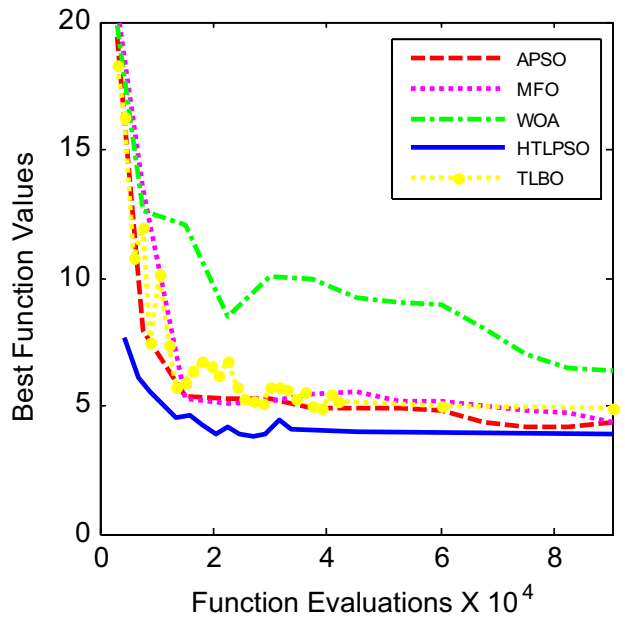

(a)

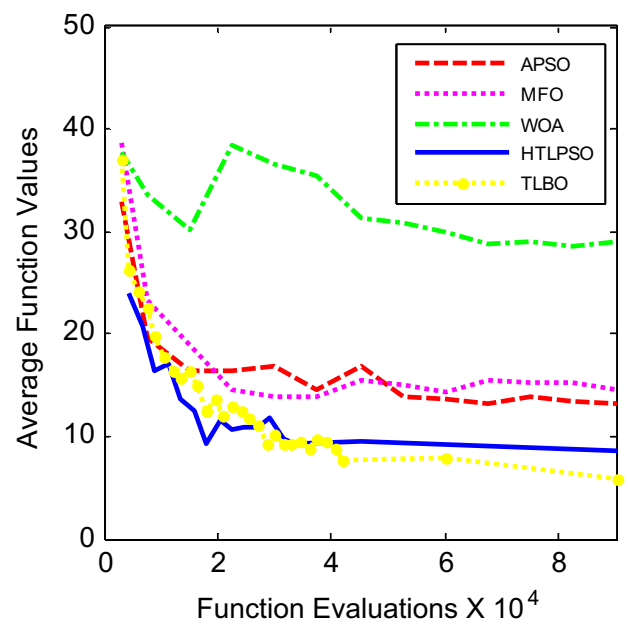

(b)

Figure 9. Convergence between (a) best function values and number of function evaluations and (b) average function values and number of function evaluations for case 2 .

Table 4. Comparison of HTLPSO with various algorithms used in literature, for case 2.

\begin{tabular}{|c|c|c|c|c|c|c|c|c|c|}
\hline $\begin{array}{l}\text { Design } \\
\text { variables }\end{array}$ & GA [8] & PSO [8] & DE [8] & TLBO & MFO & WOA & APSO & $\begin{array}{c}\text { HTRCA } \\
{[23]}\end{array}$ & HTLPSO \\
\hline$r_{1}$ & 50.00000 & 49.994859 & 50.00000 & 50.000 & 50.0000 & 49.1672 & 54.5605 & 49.46 & 50.0000 \\
\hline$r_{2}$ & 9.164414 & 5.0000000 & 5.000000 & 5.9278 & 5.0000 & 8.3843 & 8.1548 & 5.405 & 5.00000 \\
\hline$r_{3}$ & 16.85808 & 5.9156430 & 5.905345 & 9.2490 & 8.8532 & 17.7921 & 19.9956 & 8.015 & 7.31650 \\
\hline$r_{4}$ & 50.00000 & 49.994867 & 50.00000 & 50.000 & 46.4047 & 40.8881 & 45.2836 & 47.165 & 48.0298 \\
\hline$r_{c x}$ & 38.45887 & 18.925715 & 18.81931 & 25.110 & 17.9879 & 32.8107 & 40.6120 & 17.90 & 19.9172 \\
\hline$r_{c y}$ & 0.090117 & 0.0000000 & 0.000000 & -6.1922 & 20.3398 & 20.2363 & 22.7522 & 15.30 & 9.74970 \\
\hline$O_{2 x}$ & 32.32828 & 14.472475 & 14.37377 & 22.204 & 12.8609 & 29.7055 & 33.2163 & 12.00 & 14.7515 \\
\hline$O_{2 y}$ & -29.53705 & -12.49441 & -12.4442 & -17.704 & -22.0471 & -30.7715 & -38.0495 & -18.7 & -16.5078 \\
\hline$\theta_{1}$ & 0.877212 & 0.467287 & 0.463633 & 0.9123 & 6.2832 & 0.7773 & 0.7787 & 6.2832 & 0.31140 \\
\hline $\begin{array}{c}\text { Function } \\
\text { value }\end{array}$ & 3.171063 & 2.35529 & 2.349649 & 4.9439 & 4.3587 & 6.3559 & 4.2199 & 3.571 & 3.766 \\
\hline
\end{tabular}

violated and $B_{m}$ is the Boolean Function [40] expressed as follows:

$$
B_{m}= \begin{cases}0 & \text { if } g_{m}(\mathbf{x}) \leq 0 \\ 1 & \text { otherwise }\end{cases}
$$

The optimization problem formulated in Eq. (12) is solved considering three different cases: straight line, semi-circular and elliptical path generations as case 1 , case 2 and case 3 , respectively.

\section{Case 1}

This case illustrates path generation without prescribed timing for four-bar linkage [8]. In this case, the vertical straight line is to be tracked, which is composed of 6 prescribed points. The coordinates of prescribed points and limit on the design variables are as follows:

$$
\begin{aligned}
& P_{d}^{k}=[(20,20),(20,25),(20,30),(20,35),(20,40),(20,45)], \\
& r_{1}, r_{2}, r_{3}, r_{4} \in[5,60] ; r_{p x}, r_{p y}, O_{2 x}, O_{2 y} \in[-60,60] ; \theta_{1} \in[0,2 \pi] .
\end{aligned}
$$

To find out the optimal solution for this case, five algorithms are used as solvers, and their results are compared to those established algorithms in the literature [9, 19], as shown in table 3. The comparison shows that HTLPSO performs better than those of single nature-inspired algorithms, whereas HTLPSO cannot find the better solution in comparison with HTRCA. However, the difference between function values obtained through HTLPSO and HTRCA is negligible.

The rate of convergence for the best and average objective function against the number of function evaluations is shown in figure 7. Figure 8 shows the generated trajectories and prescribed points for the four-bar linkage using various optimization algorithms, for case 1 . HTLPSO 


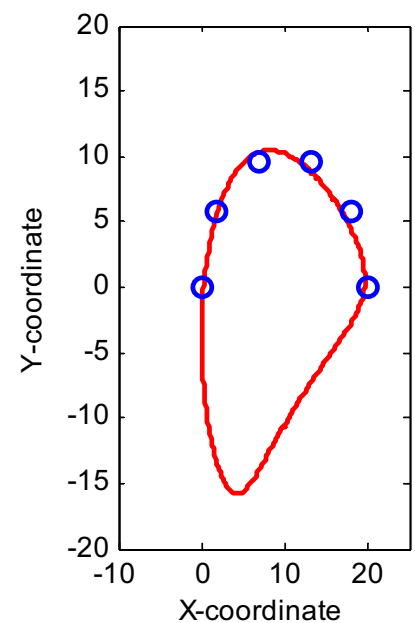

GA [14]

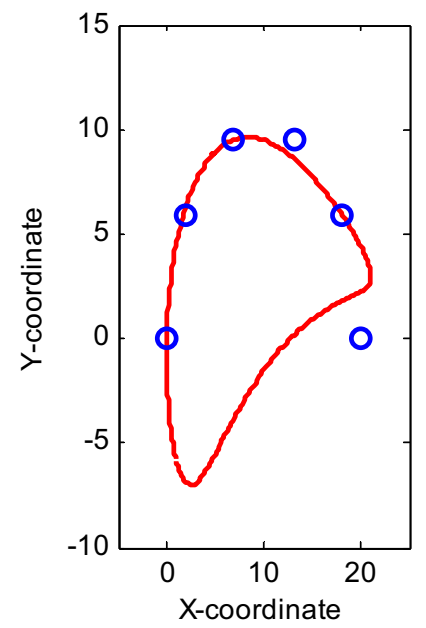

TLBO

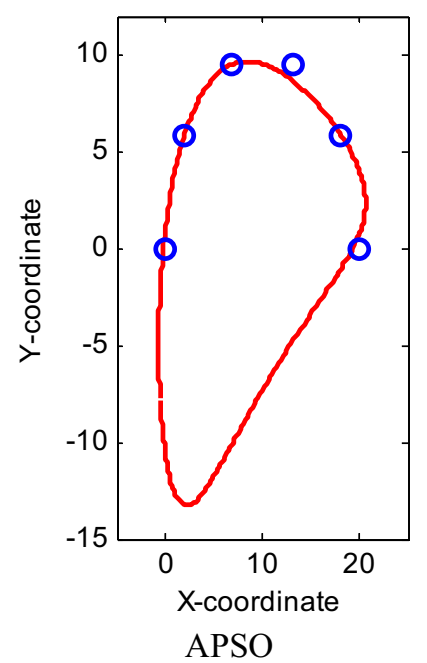

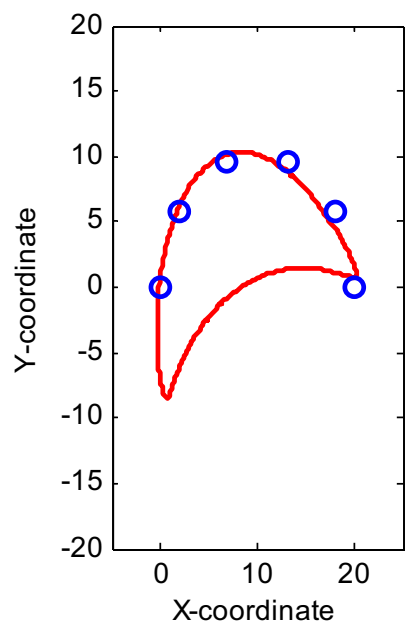

PSO [14]

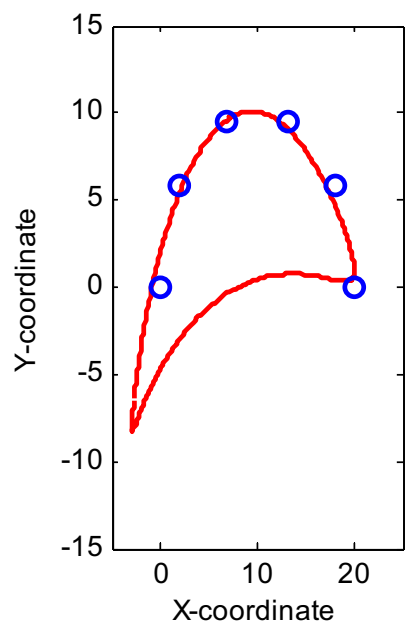

HTRCA [22]

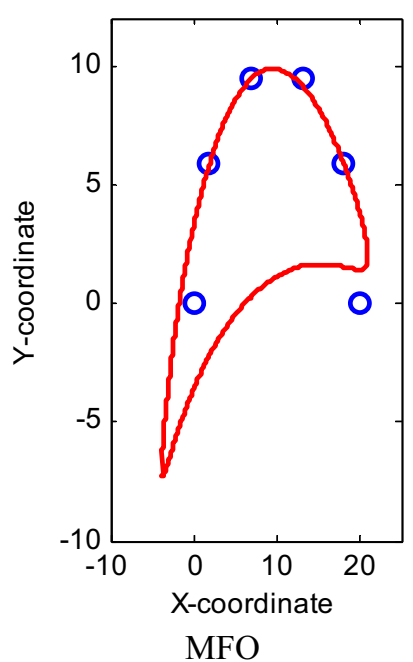

Generated Trajectory

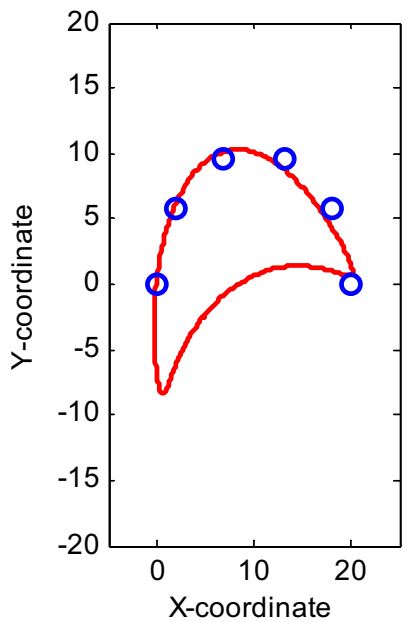

DE [14]

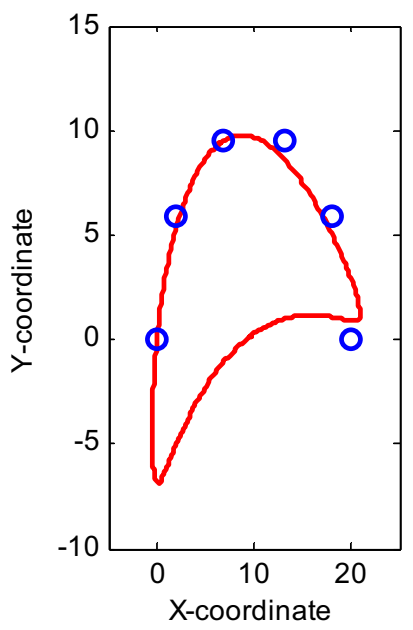

HTPLSO

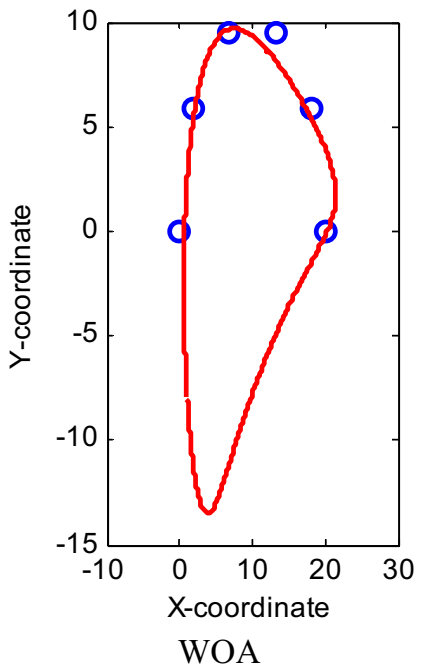

Figure 10. Generated trajectory and prescribed points. 
Table 5. Comparison of HTLPSO with various algorithms used in literature, for case 3.

\begin{tabular}{lccccccccc}
\hline Design variables & GA [8] & PSO [8] & DE [8] & TLBO & MFO & WOA & APSO & HTRCA [23] & HTLPSO \\
\hline$r_{1}$ & 79.981513 & 52.53516 & 54.36089 & 75.9859 & 80.0000 & 79.4190 & 59.9306 & 54.875 \\
$r_{2}$ & 9.1099930 & 8.687886 & 8.683351 & 8.2946 & 5.0000 & 20.9035 & 8.3158 & 9.725 & 80.0000 \\
$r_{3}$ & 72.936511 & 36.15508 & 34.31863 & 39.1410 & 63.0691 & 77.8578 & 32.2771 & 49.925 \\
$r_{4}$ & 80.000000 & 80.00000 & 79.99617 & 48.2598 & 79.9922 & 78.4010 & 44.6219 & 23.375 & 48.3073 \\
$r_{c x}$ & 0.0000000 & 0.000000 & 0.000187 & -1.4864 & -80.0000 & 58.1669 & -3.3149 & 7.8400 & 1.6448 \\
$r_{c y}$ & 0.0000000 & 1.481055 & 1.465250 & -7.4652 & -12.0492 & 77.8962 & -7.6757 & -4.320 & -10.0785 \\
$O_{2}$ & 10.155966 & 11.00212 & 10.95434 & 7.5190 & -28.7214 & 30.2928 & 6.9639 & 1.440 & 3.92080 \\
$O_{y}$ & 10.000000 & 11.09559 & 11.074534 & 17.0350 & 80.0000 & -73.7423 & 17.5758 & 12.16 \\
$\theta_{1}$ & 0.0261490 & 1.403504 & 2.12965 & 6.2831 & 0.7605 & 6.0956 & 0.0002 & 6.1135 \\
$\theta_{2}^{1}$ & 6.2831850 & 6.282619 & 6.283185 & 0.0001 & 0.0000 & 0.0000 & 0.0000 & 0.148283 & 0.18 .0160 \\
$\theta_{2}^{2}$ & 0.6007450 & 0.615302 & 0.616731 & 0.6301 & 0.0637 & 0.0000 & 0.6275 & 0.825611 & 0.7348 \\
$\theta_{2}^{3}$ & 1.3728120 & 1.305421 & 1.310254 & 1.3420 & 0.5793 & 0.0000 & 1.3088 & 1.520531 \\
$\theta_{2}^{4}$ & 2.2105750 & 2.188053 & 2.193570 & 2.0459 & 1.1008 & 0.0000 & 2.0291 & 2.236814 & 1.4363 \\
$\theta_{2}^{5}$ & 2.8626390 & 2.913049 & 2.917170 & 2.7347 & 1.5800 & 0.0000 & 2.6835 & 2.906602 & 2.1357 \\
$\theta_{2}^{6}$ & 3.4205470 & 3.499313 & 3.490746 & 3.4154 & 2.0584 & 5.9025 & 3.3994 & 3.565079 & 3.4994 \\
$\theta_{2}^{7}$ & 4.0726110 & 4.125586 & 4.132017 & 4.0876 & 3.4290 & 5.9680 & 4.0763 & 4.295185 & 4.1961 \\
$\theta_{2}^{8}$ & 4.9103730 & 4.919977 & 4.922075 & 4.7858 & 3.9938 & 6.0654 & 4.7637 & 5.021522 & 4.8998 \\
$\theta_{2}^{9}$ & 5.6824400 & 5.685021 & 5.695372 & 5.4909 & 4.5198 & 6.1583 & 5.4510 & 5.718955 & 5.5955 \\
$\theta_{2}^{10}$ & 6.283185 & 6.282323 & 6.28297 & 6.1974 & 5.0146 & 6.2832 & 6.1877 & 0.148283 & 6.2832 \\
Function value & 2.281273 & 1.971004 & 1.952326 & 0.7701 & 22.3829 & 65.3812 & 1.873 & 0.1649 & 0.3729 \\
\hline
\end{tabular}

reports the error of 0.0812 , which is better in comparison with the single nature-inspired algorithms.

Case 2

This case illustrates path generation with prescribed timing for four-bar linkage [8]. Here, four-bar linkage generates a semi-circular trajectory similar to the prescribed trajectory. The coordinates of prescribed points on the path and limit on the design variables are as follows:

$$
\begin{aligned}
& P_{d}^{k}=[(0,0),(1.9098,5.8779),(6.9098,9.5106), \\
& (13.09,9.5106),(18.09,5.8779),(20,0)], \\
& r_{1}, r_{2}, r_{3}, r_{4} \in[5,50] ; r_{p x}, r_{p y}, O_{2 x}, O_{2 y} \in[-50,50] ; \theta_{1} \in \\
& {[0,2 \pi] .}
\end{aligned}
$$

In this problem, the angle of link is provided. Therefore, there are only nine design variables:

$$
\begin{gathered}
\mathbf{x}=\left[r_{1}, r_{2}, r_{3}, r_{4}, r_{p x}, r_{p y}, O_{2 x}, O_{2 y}, \theta_{1}\right], \\
\theta_{2}^{k}=\left[\frac{\pi}{6}, \frac{\pi}{3}, \frac{\pi}{2}, \frac{2 \pi}{3}, \frac{5 \pi}{6}, \pi\right] .
\end{gathered}
$$

The objective function, Eq. (12), is minimized to get a feasible solution. Figure 9 shows the convergence for the best and average objective function with the number of function evaluations for case 2 . Table 4 shows the result comparison of proposed algorithm with the other established algorithms. The generated and prescribed trajectories of case 2 are shown in figure 10.
Case 3

This case illustrates path generation of four-bar linkage without prescribed timing. Here, totally ten prescribed positions are given to track an elliptical trajectory. The trajectory is expressed as follows.

Prescribed points:

$$
\begin{aligned}
& P_{d}^{k}=[(20,10),(17.66,15.142),(11.736,17.878), \\
& (5,16.928),(0.60307,12.736), \ldots,(0.60307,7.2638), \\
& (5,3.0718),(11.736,2.1215),(17.66,4.8577),(20,10)] .
\end{aligned}
$$

Limits of the design variables:

$$
\begin{aligned}
& r_{1}, r_{2}, r_{3}, r_{4} \in[5,80] ; r_{p x}, r_{p y}, O_{2 x}, O_{2 y} \in[-80,80] \\
& \theta_{1} \in[0,2 \pi]
\end{aligned}
$$

The results obtained using HTLPSO are compared with other algorithms and shown in table 5. Figure 11 shows the plots of the generated trajectory and prescribed points, for case 3 . The convergence of the best and average objective function is shown in figure 12. In this case, HTLPSO obtains the function value of 0.3729 , which is much better than those by single nature-inspired algorithms used here. The HTLPSO algorithm cannot find the better solution than that by the HTRCA algorithm, but the difference in values between the HTLPSO and HTRCA algorithm used here is thought to be negligible. It is observed that HTLPSO gives 


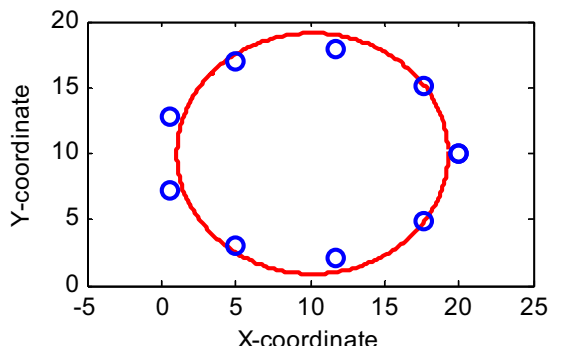

GA [14]

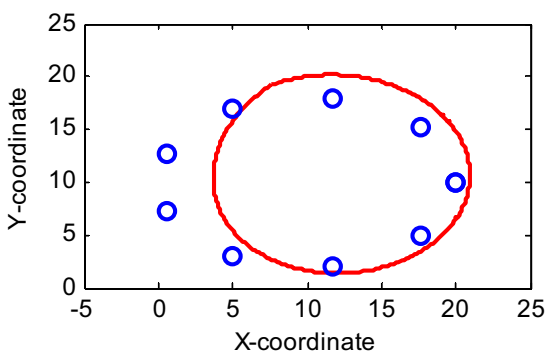

DE [14]

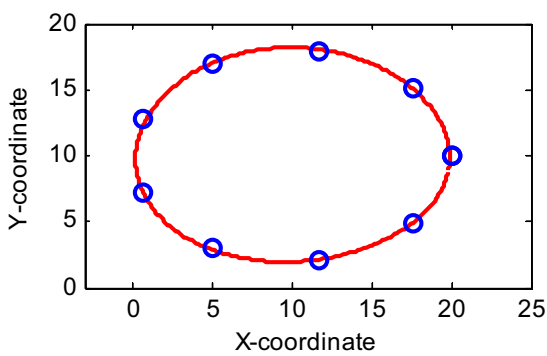

HTRCA [22]

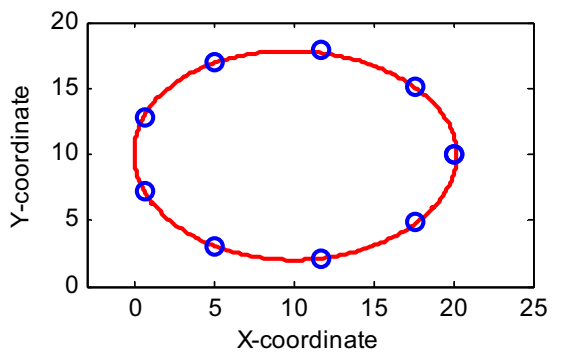

ASPO

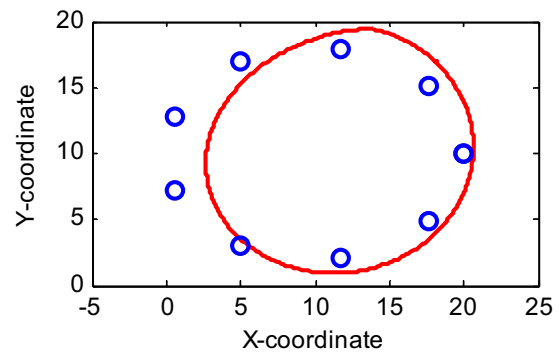

PSO [14]

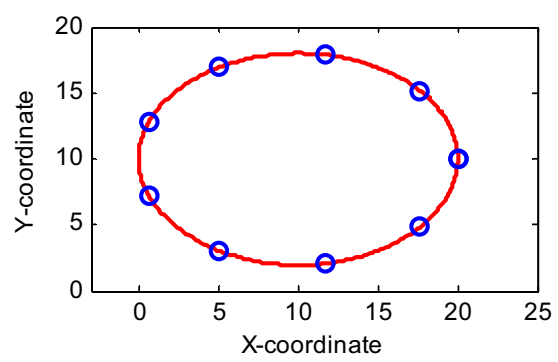

TLBO

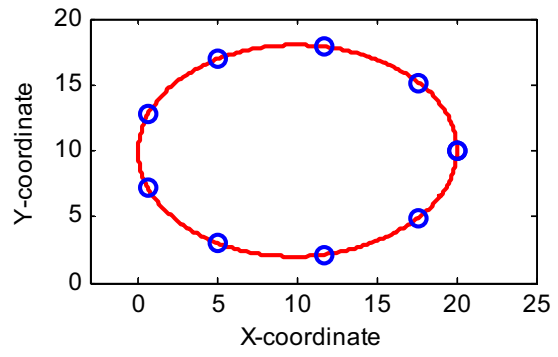

HTLPSO (proposed)

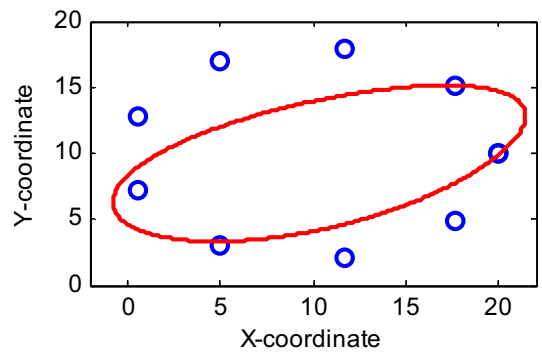

MFO

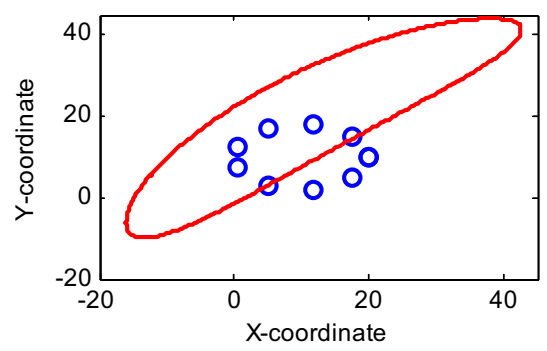

WOA

\section{Generated Trajecto
Prescribed Points}

Figure 11. Generated trajectory and prescribed points. 


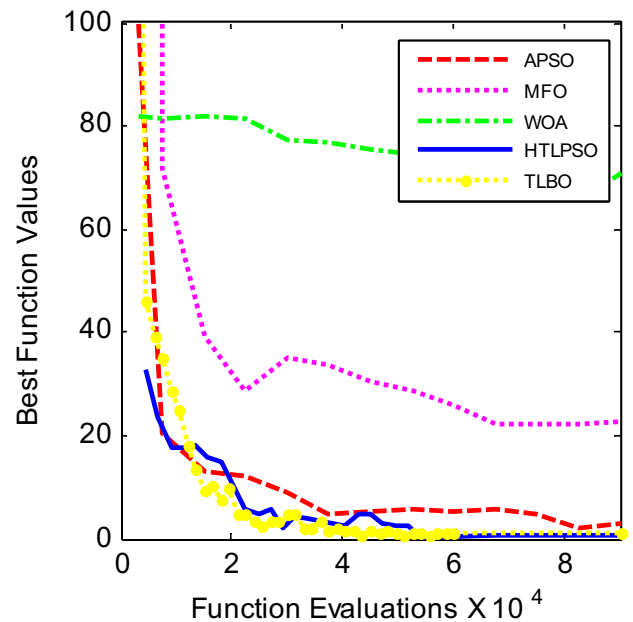

(a)

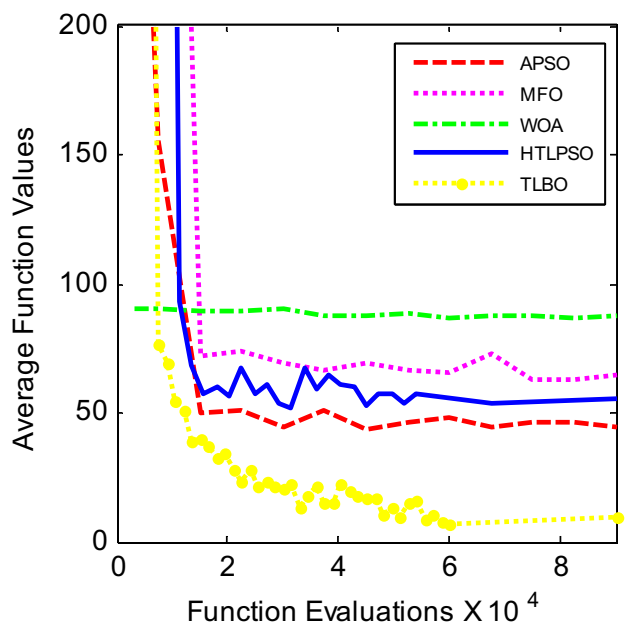

(b)

Figure 12. Convergence between (a) best function values and number of function evaluations and (b) average function values and number of function evaluations for case 3 .

Table 6. Comparison of HTLPSO with TLBO in terms of number of function evaluations.

\begin{tabular}{lccccc}
\hline & \multicolumn{5}{c}{ Number of function evaluations } \\
\cline { 2 - 6 } Case no. & HTLPSO & TLBO & WOA & MFO & APSO \\
\hline 1 & 47250 & 60000 & 90000 & 90000 & 60000 \\
2 & 24750 & 39000 & 90000 & 90000 & 75000 \\
3 & 54000 & 55500 & 82500 & 82500 & 82500 \\
\hline
\end{tabular}

the best solution compared with the other algorithms while using lesser function evaluations, as shown in table 6 .

The application of HTLPSO on these cases proved that the HTLPSO algorithm is more robust in comparison with the other algorithms used here. HTLPSO can find global optimal value in all the cases, while MFO and WOA cannot find the near approximation to global optimal solution in case 3, and the TLBO takes more function evaluations to converge. This means that merging of PSO with TLBO assists in escaping the local optima successfully. This merging has also improved the effectiveness of the algorithm. Now, HTLPSO algorithm can find optimal values for all the cases in lesser number of function evaluations for the algorithms used here. Note that the number of function evaluations of the other algorithms for the path synthesis cannot be accessed. Hence, the comparison of proposed algorithm with only APSO, TLBO, MFO and WOA in terms of the number of function evaluations is performed.

Lastly, HTLPSO can find more accurate optimal values than all the recent metaheuristic and benchmark algorithms used here. Therefore, HTLPSO can be considered as an effective algorithm for solving a wide range of problems.

The results of HTLSPO algorithm are found to be competitive and superior over the conventional and new single nature-inspired algorithms used here for comparison.
However, compared with HTRCA, the HTLPSO algorithm cannot find the better solution. Nevertheless, the difference in results of HTLSPO and HTRCA is negligible. Also, the result of HTLPSO in case 2 is worse than with other algorithms except for TLBO, WOA, MFO and APSO. The TE of all algorithms in case 2 lies in the range 2.349-4.943, which is greater than that of benchmark single-nature inspired algorithm (GA [8], PSO [8], DE [8]). However, the results are superior to the recent single nature-inspired algorithms used here (MFO, WOA). This implies that hybrid algorithms (HTRCA and HTLPSO) cannot obtain optimal solutions compared with GA [8], PSO [8] and DE [8] for the case of path synthesis with prescribed timing. From this discussion, it is found that performance of HTLPSO is superior to that of other metaheuristics used here. However, using a different operator for exploitation to improve the performance of HTLPSO in prescribed timing (Case 2) case may be the subject of future studies.

\section{Conclusions}

This paper proposed a HTLPSO using two established single nature-inspired algorithms, namely, TLBO and PSO. Both the established algorithms are also demonstrated along with a flowchart for the proposed algorithm. In this algorithm, the initial population is simultaneously fed into teacher phase of TLBO and PSO. Then, the best half of populations obtained after PSO and teacher phase are merged and resulting population is fed into learner phase. The final population obtained after learner phase is used as the initial population for PSO and teacher phase. This cycle is repeated for each iteration. To validate the proposed algorithm, five constrained benchmark functions and a path generation problem for four-bar linkage are considered. 
The WOA, MFO and APSO are among other algorithms apart from TLBO and PSO, which are used for comparing the results to HTLPSO. It is found that the proposed algorithm performs better than single nature-inspired algorithms such as PSO, MFO, WOA, APSO and TLBO for all the five benchmark functions. It also finds the better solution for path generation cases without prescribed timing. However, the HTLPSO cannot find near optimal solution for path synthesis cases with prescribed timing. However, HTLPSO finds reasonable optimal solutions in comparison with the other algorithms. Hence, HTLPSO may prove to be an important tool for a wide range of problems, including path synthesis of four-bar linkages.

\section{Acknowledgements}

Ph.D. scholarship granted by Ministry of Human Resource and Development, Government of India, to the first author is highly acknowledged.

\section{References}

[1] Norton R L 2009 Kinematics and dynamics of machinery, 1st ed. New Delhi: Tata McGraw Hill Education Private Limited

[2] Hartenberg R S and Denavit J 1964 Kinematic synthesis of linkages, 1st ed. United States of America: Tata McGraw Hill Education Private Limited

[3] Uicker J J, Pennock G R and J E.Shigley 2015 Theory of machines and mechanisms, 4th ed. India: Oxford University Press

[4] Sandor G N and Erdman A G 1984 Advanced mechanism design: analysis and synthesis, 1 st ed. United States of America: Prentice Hall

[5] Roth B and Freudenstein F 1963 Synthesis of path-generating mechanism by numerical methods. Trans. ASME J. Eng. Ind. 85(3): 298-304

[6] Kunjur A and Krishnamurty S 1997 Genetic algorithms in mechanism synthesis. J. Appl. Mechan. Robot. 4(2): 18-24

[7] Nishad S S, Dutta A and Saxena A 2014 Design and control of a three finger hand exoskeleton for translation of a slender object. In: Proceedings of the $11^{\text {th }}$ IEEE International Conference on Ubiquitous Robots and Ambient Intelligence, Kuala Lumpur, Malaysia, November 12-15, pp. 179-184

[8] Acharyya S K and Mandal M 2009 Performance of EAs for four-bar linkage synthesis. Mech. Mach. Theory 44(9): 1784-1794

[9] Ettefagh M M, Abbasi M and Emdadi H 2013 Path synthesis of four-bar mechanism using $\mathrm{ABC}$ algorithm and comparing with BGA. In: Proceedings of the IEEE Symposium on Innovations in Intelligent Systems and Applications, Albena, June 19-21, pp. 1-5

[10] Han C Y 1967 A general method for the optimum design of mechanisms. J. Mech. 1(3): 301-313

[11] Zadeh N N, Felezi M, Jamali A and Ganji M 2009 Pareto optimal synthesis of four-bar mechanisms for path generation. Mech. Mach. Theory 44(1): 180-191
[12] Khorshidi M, Soheilypour M, Peyro M, Atai A and Panahi M S 2011 Optimal design of four-bar mechanisms using hybrid multi-objective GA with adaptive local search. Mech. Mach. Theory 46(10): 1453-1465

[13] Mariappan J and Krishnamurty S 1996 A generalized exact gradient method for mechanism synthesis. Mech. Mach. Theory 31(4): 413-421

[14] Zhang X, Zhou J and Ye Y 2000 Optimal mechanism design using interior-point methods. Mech. Mach. Theory 35(1): 83-98

[15] Sancibrian R, Garcia P, Viadero F and Fernandez A 2006 A general procedure based on exact gradient determination in dimensional synthesis of planar mechanisms. Mech. Mach. Theory 41(2): 212-229

[16] Mirjaali S and Lewis A 2016 The whale optimization algorithm. Adv. Eng. Softw. 95: 51-67

[17] Mirjaali S 2015 Moth-flame optimization algorithm: a novel nature-inspired heuristic paradigm. Knowl.-Based Syst. 89: 228-249

[18] Martinez-Alfaro H 2007 Four-bar mechanism synthesis for $n$ desired path points using simulated annealing. In: Siarry $\mathrm{P}$ and Michalewicz Z (Eds.) Advances in metaheuristics for hard optimization. Springer Berlin, pp. 23-27

[19] Cabrera J A, Simon A and Prado M 2002 Optimal synthesis of mechanisms with genetic algorithms. Mech. Mach. Theory 37(10): 1165-1177

[20] Rao R V, Savsani V J and Vakharia D P 2011 Teachinglearning-based optimization: a novel method for constrained mechanical design optimization problems. Comput.-Aided Des. 43(3): 303-315

[21] Lai X and Zhang M 2009 An efficient ensemble of GA and PSO for real function optimization. In: Proceedings of the 2nd IEEE International Conference on Computer Science and Information Technology, ICCSIT, Beijing, August 8-11, pp. 651-655

[22] Lin W Y 2010 A GA-DE hybrid evolutionary algorithm for path synthesis of four-bar linkage. Mech. Mach. Theory 45(8): 1096-1107

[23] Kim J W, Jeong S M, Kim J and Seo T 2016 Numerical hybrid Taguchi-random coordinate search algorithm for path synthesis. Mech. Mach. Theory 102: 203-216

[24] Soleimani H and Kannan G 2015 A hybrid particle swarm optimization and genetic algorithm for closed-loop supply chain network design in large-scale networks. Appl. Math. Model. 39: 3990-4012

[25] Yogesh C K, Hariharan M, Ngadiran R, Adom A H,Yaacob S, Berkai C and Polat K 2017 A new hybrid PSO assisted biogeography-based optimization for emotion and stress recognition from speech signal. Expert Syst. Appl. 69: 149-158, DOI:http://dx.doi.org/10.1016/j.eswa.2016.10.035

[26] De A, Mamanduru V K R, Gunasekaran A, Subramanian N and Tiwari M K 2016 Composite particle algorithm for sustainable integrated dynamic ship routing and scheduling optimization. Comput. Ind. Eng. 96: 201-215

[27] De A, Awasthi A and Tiwari M K 2015 Robust formulation for optimizing sustainable ship routing and scheduling problem. IFAC-PapersOnLine 48(3): 368-373, DOI:http:// doi.org/10.1016/j.ifacol.2015.06.109

[28] De A, Kumar S K, Gunasekaran A and Tiwari M K 2017 Sustainable maritime inventory routing problem with time 
window constraints. Eng. Appl. Artif. Intell. 61: 77-95, DOI:http://dx.doi.org/10.1016/j.engappai.2017.02.012

[29] Pathak V K, Singh A K, Singh R and Chaudhary H 2017 A modified algorithm of Particle Swarm Optimization for form error evaluation. Tech. Mess. 84(4): 272-292, DOI:http://doi. org/10.1515/teme-2016-0040

[30] Masrom S, Moser I, Montgomery J and Abidin S Z Z 2013 Hybridization of particle swarm optimization with adaptive genetic algorithm operators. In: Proceedings of the 13th IEEE International Conference on Intelligent Systems Design and Applications (ISDA), December 8-10, DOI:10. 1109/ISDA.2013.6920726

[31] Santra D, Sarker K, Mukherjee A and Chatterjee D 2016 Hybrid PSO-ACO algorithm to solve economic load dispatch problem with transmission loss for small scale power system. In: Proceedings of the 2016 International Conference on Intelligent Control Power and Instrumentation (ICICPI), 21-23 October, pp. 226-230, DOI:http://doi.org/ 10.1109/ICICPI.2016.7859707

[32] Alexandridis A, Chondrodima E and Sarimveis H 2016 Cooperative learning for radial basis function networks using particle swarm optimization. Appl. Soft Comput. 49: 485-497
[33] Wolpert D H and Macread W G 1997 No free lunch theorems for optimization. IEEE Trans. Evol. Comput. 1(1): $67-82$

[34] Gao H and Xu W 2011 Particle swarm algorithm with hybrid mutation strategy. Appl. Soft Comput. 11(8): 5129-5142

[35] Rao R V and Savasani V J 2012 Mechanical design optimization using advanced optimization techniques. London: Springer Science \& Business Media

[36] Eiben A E, Hinterding R and Michalewicz Z 1999 Paramater control in evolutionary algorithms. IEEE Trans. Evol. Comput. 3(2): 124-141

[37] Dong Y, Tang J, Xu B and Wang D 2005 An application of swarm optimization to nonlinear programming. Comput. Math. Appl. 49(11-12): 1655-1668

[38] Rao R V 2015 Teaching learning based optimization algorithm and its engineering applications. Springer, Switzerland

[39] Rao R V and Patel V 2012 An elitist teaching-learning optimization algorithm for solving complex constrained optimization problems. Int. J. Ind. Eng. Comput. 3: 535-560

[40] Mundo D, Gatti G and Dooner D B 2009 Optimized five-bar linkages with non-circular gears for exact path generation. Mech. Mach. Theory 44(4): 751-760 